\title{
Molecular Dynamics Study of the Interface Between Water and 2-Nitrophenyloctyl Ether
}

\author{
Miguel Jorge*, M. Natália D. S. Cordeiro* \\ REQUIMTE, Faculdade de Ciências, Universidade do Porto, Rua do Campo Alegre, 687, 4169- \\ 007 Porto, Portugal
}

Email addresses: miguel.jorge@fc.up.pt; ncordeir@fc.up.pt

Title Running Head: Molecular dynamics of Water/NPOE interface

\begin{abstract}
We present results of molecular dynamics simulations of the interface between water and 2nitrophenyloctyl ether (NPOE). This system is analyzed in detail using a procedure to calculate intrinsic profiles of several important properties (density, radial distribution functions, hydrogen bonds, molecular orientation, self-diffusion). The interface was found to be molecularly sharp, but corrugated by thermal fluctuations. Using a method based on capillary wave theory, we have estimated the interfacial tension and obtained good agreement with values calculated from the virial route. The results were compared to simulations of the water/nitrobenzene interface. The presence of an alkyl chain in NPOE introduces an added degree of hydrophobicity, which causes an increase in the interfacial tension. Furthermore, interfacial NPOE molecules are less organized than nitrobenzene and show a distinct dynamic response. These results shed light on the observed differences between these two organic liquids in electrochemical studies.
\end{abstract}


Key words: liquid/liquid interface; surface tension; ion transfer; interfacial width.

\section{Introduction}

Liquid/liquid interfaces have attracted a great deal of interest from the scientific community, mostly due to their presence in many chemical, physical and biological processes, such as phase transfer catalysis, liquid-liquid extraction and drug delivery ${ }^{1}$. One particular area of enormous importance is that of molecule transfer across water/organic interfaces ${ }^{2}$. A crucial aspect controlling the efficiency of these transfer processes is the choice of organic solvent. The most widely used solvents for this purpose are $n$-octanol, 1,2-dichloroethane and nitrobenzene $(\mathrm{NB})^{3}$. The latter has a low miscibility with water and is simultaneously a good medium for ion solvation, due to its high dielectric constant. However, its use is limited by a relatively high toxicity. Recently, 2-nitrophenyloctyl ether (NPOE) has emerged as a promising alternative to the solvents mentioned above, and has shown great potential in ion-transfer studies across water/organic interfaces ${ }^{4-6}$. NPOE shares some of the advantages of NB, but toxic effects on human beings are not known to occur. Recently, our group has presented the first molecular simulation studies of NPOE, one focusing on the pure liquid properties and on ion solvation ${ }^{7}$, and another one examining the interactions of NPOE with dissolved water molecules ${ }^{8}$. However, in order to better understand molecule transfer processes at liquid/liquid interfaces, it is necessary to gather fundamental knowledge of the molecular-level properties of the interface itself. In this paper, we perform detailed molecular dynamics (MD) simulations of the water/NPOE interface with the aim of characterizing the local structure and dynamics of this interface. The results are compared to experimental data, where available, and to the properties of the water/nitrobenzene interface.

Molecular simulations are able to provide molecular-level information about interfacial systems in a way that is not generally possible using experimental techniques ${ }^{2}$. This is caused mainly by the fluidity of the interface and by its buried nature, which precludes local 
experimental probing. Thus, many studies have been devoted to simulations of liquid/liquid interfaces (see review by Benjamin ${ }^{2}$ and references therein). Some of the most insightful results regarding these systems have been obtained in early studies of water/organic interfaces. In particular, Linse ${ }^{9}$ and Benjamin ${ }^{10}$ conclusively established a picture of an interface that is relatively sharp on the molecular level, but is broadened by thermal fluctuations. They reached this conclusion by dividing the plane parallel to the interface in square meshes of different resolutions and then examining the variations in interfacial width ${ }^{9,10}$ and position ${ }^{10}$ with degree of mesh refinement. This picture was confirmed in nearly all subsequent simulation studies of realistic liquid/liquid interfaces, and led to attempts to model these systems using capillary wave theory $(\mathrm{CWT})^{11,12}$. Several authors ${ }^{10,13-18}$ have attempted to calculate the interfacial tension based on the original form of CWT, but quantitative agreement with experiment and with values calculated from the simulations by alternative routes was not always obtained.

The presence of the interface induces anisotropies in structural and dynamic properties in the direction normal to the interfacial plane. The most obvious example of this is the density profile, which exhibits a transition from one bulk density to the other across a relatively narrow interfacial region ${ }^{9,10,13-21}$. Other properties have been calculated for several water/organic interfaces, including radial distributions functions (RDFs) ${ }^{10,15,17,19}$, hydrogen bonds ${ }^{9,10,13-15,17-19,21}$, molecular orientations $s^{9,10,13-15,17-24}$ and self-diffusion coefficients ${ }^{10,13-15,23}$. For this purpose, the simulation box is usually divided in slices parallel to the interfacial plane and properties are computed as a function of distance to the interface. In most cases, the interface is defined globally, i.e., it is assumed to be a plane located somewhere between the two bulk phases (the exact location of this plane is itself a subject of debate). Although it is able to shed some light into the way bulk phases are affected by the interface, this method of calculation is unable to provide a detailed description of the intrinsic properties of the interfacial system. The reason for this is that the liquid/liquid interface is normally not flat; instead, as discussed above, it is 
corrugated by capillary waves. Thus, properties calculated relative to a global definition of the interface are subject to smoothing caused by fluctuations of the interfacial plane.

In an effort to circumvent this problem, Fernandes et al. ${ }^{16}$ have proposed a procedure for decoupling fluctuations of the interfacial plane from those occurring perpendicularly to the interface. Using this method, they calculated density profiles that showed pronounced oscillations in the vicinity of the interface, which died out as the bulk phases were approached. Their method was later applied to other liquid/liquid interfaces ${ }^{17,18}$, but it was not conclusively established that true intrinsic profiles were obtained. Tarazona and Chacón ${ }^{25,26}$ developed a self-consistent method for the identification of the interfacial plane as the minimal-area surface going through a set of pivot atoms. They have applied it to calculate intrinsic density profiles of several liquid/vapor interfaces ${ }^{25-28}$. These advances have prompted Chowdhary and Ladanyi ${ }^{29}$ to adapt their procedure to water/alkane interfaces. As well as density profiles, those authors also computed molecular orientation profiles as a function of distance to the locally defined interface. In a recent paper $^{30}$, we have modified the method of Fernandes et al. ${ }^{16}$ by extending it to atomic resolution. In so doing, we were able to obtain true intrinsic density profiles, as well as several other intrinsic properties, for the water/NB interface, thus conclusively establishing a detailed molecular-level description of this interface. In the present article, we apply the same methodology to the water/NPOE interface. The paper is organized as follows: Section 2 provides details of the theoretical methods employed, including potential models, simulation details, local interface definition, calculation of the interfacial tension, molecular orientations and diffusion coefficients; our results are presented and discussed in Section 3; conclusions are given in Section 4.

\section{Computational Details}

\subsection{Potential Models}


Water molecules were represented by the SPC/E potential ${ }^{31}$ with rigid bonds and angle. NPOE and NB were modeled by the OPLS all-atom transferable potential ${ }^{32,33}$ with rigid bonds, flexible angles and flexible dihedrals. The resulting potential energy is thus the sum of harmonic angle-bending terms, proper and improper torsional terms, Lennard-Jones and Coulomb electrostatic terms. Parameters for the organic liquids were reported in a previous publication ${ }^{7}$. A twin range cutoff scheme was employed to calculate the short-range dispersion interactions, with cutoff radii of 1.1 and $1.5 \mathrm{~nm}$. A long-range dispersion correction was added to both energy and pressure. Long-range electrostatics were handled using the particle-mesh Ewald method ${ }^{34}$ with a real-space cutoff of $1.1 \mathrm{~nm}$. A diagram depicting the molecular structure of NB and NPOE, including the nomenclature given to each atom type, is shown in Figure 1.

\section{Figure 1}

\subsection{Simulation Methods}

We have performed MD simulations in the $N P_{\mathrm{z}} A T$ ensemble ${ }^{20}$ using GROMACS $3.3^{35,36}$. Equations of motion were integrated with the Verlet leapfrog algorithm ${ }^{37}$ and a time step of 2 fs. Constraints on the bond lengths were enforced using the LINCS algorithm ${ }^{38}$. The chosen ensemble requires keeping the number of molecules $(N)$, the temperature $(T)$, the cross-sectional area $(A)$ and the pressure normal to the interfacial plane $\left(P_{\mathrm{z}}\right)$ constant during the simulation run. The Nosé-Hoover thermostat ${ }^{39,40}$ was applied to control the temperature at $298 \mathrm{~K}$, while the normal pressure was kept at 1 bar using the semi-isotropic form of the Parrinello-Rahman barostat $^{41}$. The interfacial systems were constructed by first equilibrating two separate boxes containing the water and organic liquids with the same cross-sectional dimensions. These boxes were then fitted together leaving a small gap between them in order to avoid atomic overlap. A translation of the entire system in the direction perpendicular to the interface was performed until the center of mass of the organic phase was located in the middle of the simulation box. The resulting simulation box was a cuboid, elongated in the direction perpendicular to the interface 
$\left(L_{\mathrm{z}}>L_{\mathrm{x}}=L_{\mathrm{y}}=L\right) . L_{\mathrm{x}}$ and $L_{\mathrm{y}}$ were kept fixed, keeping the cross-sectional area constant, while $L_{\mathrm{z}}$ was allowed to fluctuate. Periodic boundary conditions were applied in all directions, giving rise to two separate interfaces. The system was equilibrated until $L_{\mathrm{z}}$ oscillated around a constant value (typically after about 500 ps), and properties were then sampled during 5 ns. The sampling periods were divided into blocks of 200 ps for averaging purposes. We have performed a single simulation run for water/NB, with $L=3.5 \mathrm{~nm}$ and $L_{\mathrm{z}} \approx 8.5 \mathrm{~nm}$, which included $296 \mathrm{NB}$ molecules and 1757 water molecules. For water/NPOE, we have performed simulation runs with $L=3.5 \mathrm{~nm}$ and $L=4.5 \mathrm{~nm}$, in both cases with $L_{\mathrm{z}} \approx 10 \mathrm{~nm}$. The former included $180 \mathrm{NPOE}$ molecules and 1757 waters, while the latter included 270 NPOE molecules and 3007 waters. All results shown here were observed to be independent of the value of $L$ within the chosen range.

\subsection{Global and Intrinsic Properties}

Global properties of the interfacial system were calculated relative to the interface location defined in terms of a fixed coordinate frame. In other words, the interface was defined globally as a plane residing halfway between the bulk water and organic phases. Global properties were computed for each configuration of a given MD run using the following procedure. First, the $z$ coordinates of each site were rescaled by a fixed amount so as to place the center of mass of the organic phase at the origin, thus eliminating artifacts due to possible translational drift of the interface ${ }^{10}$. Then, the simulation box was divided in the $z$ direction into slabs of $0.04 \mathrm{~nm}$ width. Finally, the instantaneous value of the property of interest for each species was calculated in each slab. Averaging over the whole sampling period yields the global profile of the chosen property.

Intrinsic properties, on the contrary, were calculated relative to a local definition of the interfacial plane. More precisely, a procedure was employed to locate the limits $(l)$ of each phase (water and organic) at the finest possible resolution, and properties in the opposite phase were then computed relative to this location. To achieve this, we divided the $x y$ plane of the simulation 
box in a square mesh of a pre-defined resolution, given by an integer parameter $N$. We are thus left with $N^{2}$ prisms of size $\frac{L_{\mathrm{x}}}{N} \times \frac{L_{\mathrm{y}}}{N} \times L_{\mathrm{z}}$. In each of these prisms, we have located the limits of the water (organic) phase, defined as the $z$ coordinate of the water (organic) site that protruded furthest into the organic (water) phase but was still linked to the bulk water (organic) phase. This definition excludes overhangs and dissolved molecules from the calculation of the interface location. Once this was done, the chosen property was computed separately in each prism, by dividing it into $0.04 \mathrm{~nm}$ slabs, relative to these limits. Averaging over all prisms and all configurations of the sampling period yields the average profile of the property of interest at the chosen value of $N$.

The procedure described in the previous paragraph is similar to the one proposed by Fernandes et al. ${ }^{16}$ for the calculation of intrinsic density profiles. However, as demonstrated in our previous publication ${ }^{30}$, those authors employed a value of $N$ that was too low to obtain true intrinsic profiles. Their choice was based on the derivation by Weeks ${ }^{42}$ of a capillary wave Hamiltonian, which imposed $L / N>\xi$, where $\xi$ is the bulk correlation length of the fluid. This variable is normally taken to be of the order of molecular size, following the argument that “capillary waves" are no longer meaningful below molecular dimensions. However, Tarazona and Chacón ${ }^{25-27}$ have shown that one must go beyond the limits of applicability of CWT in order to calculate true intrinsic profiles. More precisely, the resolution for defining the interfacial plane should be close to the value of the Lennard-Jones site diameter $(\sigma)$ of the liquid ${ }^{26}$. Only at this resolution is the profile free from the influence of thermal fluctuations of the interface position. Here, we adopt this criterion for choosing the optimal value of $N$ in order to obtain true intrinsic profiles across the interface. Thus, for a given component (water, NB or NPOE), $N$ was chosen such that $L / N$ was approximately equal to the diameter of the largest atomic site of the opposite phase. The largest site of the SPC/E model is $\sigma=0.31656 \mathrm{~nm}$ and the largest site of both the NB 
and NPOE models (according to the OPLS potential) is $\sigma=0.355 \mathrm{~nm}$. Therefore, for $L=3.5 \mathrm{~nm}$ the water intrinsic profiles were obtained at $N=10$ and the organic intrinsic profiles at $N=11$, while for $L=4.5 \mathrm{~nm}$ the water and organic profiles were calculated at $N=13$ and $N=14$, respectively. Further details regarding the choice of $N$ as well as the implementation of the calculation procedure were given in a previous publication ${ }^{30}$.

By determining the limits of each phase, it is also possible to calculate the interface position $(h)$ and width $(w)$ in each prism. This is done using the following equations:

$$
\begin{aligned}
& h_{\mathrm{L}}=\frac{l_{\mathrm{WL}}+l_{\mathrm{OL}}}{2} \\
& h_{\mathrm{R}}=\frac{l_{\mathrm{WR}}+l_{\mathrm{OR}}}{2} \\
& w_{\mathrm{L}}=l_{\mathrm{WL}}-l_{\mathrm{OL}} \\
& w_{\mathrm{R}}=l_{\mathrm{OR}}-l_{\mathrm{WR}}
\end{aligned}
$$

The subscripts $L, R, W$ and $O$ are for the left interface, the right interface, the water phase and the organic phase, respectively. One can then average $h$ and $w$ over all prisms and all sampled configurations to obtain distributions of these properties for the chosen value of $N$. A very similar procedure was previously employed by Linse $^{9}$ and by Benjamin ${ }^{10}$ to demonstrate the sharp and corrugated nature of the water/organic interface.

\subsection{Interfacial Tension}

In this paper, the interfacial tension $(\gamma)$ was calculated from the simulations using two independent methods. The first is the so-called virial route, which is based on a relationship between $\gamma$ and the integral of the components of the pressure tensor over the whole simulation box. The expression for calculating the surface tension in our systems is ${ }^{12}$ :

$$
\gamma_{\mathrm{v}}=\frac{1}{2}\left\langle L_{\mathrm{z}}\left(P_{\mathrm{zz}}-\frac{P_{\mathrm{xx}}+P_{\mathrm{yy}}}{2}\right)\right\rangle
$$


where $P_{\mathrm{ij}}$ is the $i j$ component of the pressure tensor, the factor $1 / 2$ is due to the presence of two interfaces and the angle brackets represent an ensemble average. The box length in the direction normal to the interface is included in the ensemble average since it is allowed to vary. This method permits the direct calculation of the interfacial tension from the simulated trajectory, but normally suffers from large statistical errors due to fluctuations of the pressure tensor components. However, as we will see below, our simulation runs are long enough to allow statistically meaningful results to be obtained.

The other method for calculating $\gamma$ is based on CWT, more precisely on a relation between the interfacial tension and the width of the interface due to capillary wave fluctuations $\left(w_{\mathrm{cw}}\right)$ :

$$
w_{\mathrm{cw}}^{2}=\frac{k_{\mathrm{B}} T}{2 \pi \gamma_{\mathrm{cw}}} \ln \left(\frac{L}{\xi}\right)
$$

where $k_{\mathrm{B}}$ is the Boltzmann constant. This expression neglects the effects of gravity, which is a reasonable assumption given the small length scale of the simulations ${ }^{2}$. In order to calculate $\gamma$, one must obtain an estimate of the capillary wave width and of the bulk correlation length. This is not straightforward. Several previous studies $^{10,13-18}$ have computed the total width of the interface, usually from the mean square deviation of the interface position, and applied it directly in equation (6) together with rough estimates of $\xi$ (ranging from 0.4 to $0.9 \mathrm{~nm}$ ). The rather loose interpretation of these parameters has led to frequent disagreement with interfacial tensions calculated by the virial route.

An important breakthrough in the modeling of liquid/liquid interfaces by CWT has been put forward by Senapati and Berkowitz ${ }^{43}$. They have made use of a model proposed by Weeks ${ }^{42}$, which describes the interface as consisting of an intrinsic component, with an associated density profile and width, over which capillary waves are superimposed. Therefore, the total interfacial width $\left(w_{\mathrm{t}}\right)$ may be defined as the squared sum of the capillary wave width and the intrinsic width $\left(w_{\mathrm{i}}\right)$ : 


$$
w_{\mathrm{t}}^{2}=w_{\mathrm{i}}^{2}+w_{\mathrm{cw}}^{2}
$$

In the original form of CWT, it was assumed that the interface was perfectly flat in the absence of capillary waves ${ }^{11}$. Thus, a step function was taken for the intrinsic density profile, implying an intrinsic width of zero. This has led to the direct use of $w_{\mathrm{t}}$ in equation (6) in previous studies. However, several theoretical and experimental studies have demonstrated the existence of a smooth intrinsic density profile with a finite width ${ }^{25-30,44}$.

It was shown by Sides et $a l^{45}$ that equation (7) is exact when the total and intrinsic widths are obtained from the square root of the variance of the global and intrinsic density profiles, respectively. Here, we adopt their approach and compute $w_{\mathrm{t}}$ and $w_{\mathrm{i}}$ by fitting the global and intrinsic density profiles to appropriate mathematical expressions. More precisely, we fit the profiles to an error function form (the form used by Sides et $a .^{45}$ ) with a superimposed exponentially damped oscillation ${ }^{46}$ :

$$
\rho(z)=\frac{\rho_{\mathrm{B}}}{2}\left[1+\operatorname{erf}\left(\frac{z}{\sqrt{2} w_{\mathrm{e}}}\right)\right]\left[1+A \exp (B z) \cos \left(\frac{2 \pi}{C} z\right)\right]
$$

where $\rho(z)$ is the $z$-dependent density, $\rho_{\mathrm{B}}$ is the equilibrium bulk density, $w_{\mathrm{e}}, A, B$ and $C$ are fitting parameters. We have chosen to keep $\rho_{\mathrm{B}}$ fixed at the equilibrium value of the pure component densities in order to reduce the number of free parameters. Parameter $w_{\mathrm{e}}$ is related to the width of the profile, $A$ controls the amplitude of the oscillations, $B$ controls their decay rate and $C$ is the oscillation period. Equation (8) is able to satisfactorily capture the oscillations present in the density profiles calculated at water/organic interfaces ${ }^{30}$. The variance of the profile is given by:

$$
w_{\mathrm{o}}^{2}=w_{\mathrm{e}}^{2}(1+A)
$$

Another advance introduced by Senapati and Berkowitz ${ }^{43}$ was a method to calculate the interfacial tension without the need for an assumed value of the bulk correlation length. This 
method requires that at least two simulations with boxes of different cross-sectional areas be performed for the same system. The interfacial tension may then be calculated from:

$$
\gamma_{\mathrm{cw}}=\frac{k_{\mathrm{B}} T}{2 \pi\left(w_{\mathrm{cw}, I}^{2}-w_{\mathrm{cw}, I I}^{2}\right)} \ln \left(\frac{L_{I}}{L_{I I}}\right)
$$

This equation is obtained by applying equation (6) to each simulation (denoted by subscripts $I$ and $I I$ ), and combining both expressions to eliminate $\xi$ (assuming that this value does not depend on the box size). The bulk correlation length itself can then be extracted from equation (6) using the value of $\gamma_{\mathrm{cw}}$ calculated in equation (10).

In summary, the procedure employed here to calculate the interfacial tension from CWT is as follows: i) perform two simulations with different values of $L$; ii) in each simulation, calculate the global and intrinsic density profiles using the procedure described in section 2.3; iii) fit those profiles to equation (8) and calculate their variances from equation (9); iv) substitute the squared total width (variance of the global profile) and the squared intrinsic width (variance of the intrinsic profile) in equation (7) to compute the width due to capillary wave fluctuations; v) use $w_{\mathrm{cw}}$ for each simulation in equation (10) to calculate the interfacial tension; vi) use equation (6) with the calculated value of $\gamma_{\mathrm{cw}}$ to obtain the bulk correlation length.

\subsection{Hydrogen Bonds and Radial Distribution Functions}

We have calculated global and intrinsic profiles for the number of hydrogen bonds per molecule formed between two different water molecules. Furthermore, profiles for bonds formed between hydrogen atoms of water and oxygen atoms in the nitro group of NB or NPOE were also computed, as these were observed to take place in these systems ${ }^{8}$. Two molecules were considered to form a hydrogen bond if their oxygen and hydrogen atoms were located at a distance below $0.24 \mathrm{~nm}$ (i.e., up to the first minimum of the O-H RDF in bulk water). Additionally, we have calculated a profile for the total number of neighbors in the first water coordination shell. This quantity was defined as the number of water molecules whose center of 
mass was located within a radius of $0.35 \mathrm{~nm}$ (i.e., up to the first minimum of the O-O RDF in bulk water) from the central water molecule. The procedure for calculating these profiles was analogous to the one described in section 2.3, except that, in order to achieve better statistics, slabs of $0.2 \mathrm{~nm}$ were used to divide the system in the $z$ direction.

Radial distribution functions between pairs of atoms belonging to the same species were calculated as a function of distance to the interface using the same slices as for the hydrogen bond profiles. The RDF in slice $i$ between a reference site $a$ and another site $b$ was built as a distribution of distances between all sites $b$ (in all slices) and each of the reference sites $a$ whose $z$ coordinates fall within the limits of slice $i$, normalized by the average number density of those sites in the bulk phase. RDFs between sites belonging to different species (cross-species RDFs) were also computed. These were calculated in the interfacial region alone, and were normalized by the average number density of reference sites in the bulk phase.

\subsection{Molecular Orientation}

The simplest procedure for studying the orientation of molecules at interfaces is based on calculating a profile for the average value of some characteristic angle as a function of distance to the interface. In most cases, the angle between the dipole vector of the water molecule and the interface normal was chosen as the characteristic angle ${ }^{9,20,47}$. A method that yields more information involves calculating the distribution of this characteristic angle in slices perpendicular to the interface. This method has been applied to the angle between the interface normal and the water dipole vector ${ }^{10,13-15,17-21,23}$, the water $\mathrm{H}-\mathrm{H}$ vector ${ }^{10}$ and the water $\mathrm{O}-\mathrm{H}$ vector $^{14,17,19,21}$. In some studies, the procedure was also applied to organic molecules ${ }^{9,10,13-15,17-19}$. Naturally, by computing the distribution of the angle instead of simply its average value, a more detailed description of the water orientation was obtained. Nevertheless, as pointed out by Jedlovszky et al. ${ }^{22}$, monovariate distributions of angles are insufficient to completely describe the orientation of the water molecules. Instead, one must employ a bivariate angle distribution, which 
uniquely defines the orientation of a given water molecule ${ }^{22}$. In this paper, we apply Jedlovszky's method to determine the orientation of both water and organic molecules as a function of distance to the interface, using both a global and a local definition of the interfacial plane. The global and intrinsic bivariate angle distributions were calculated in slices of $0.2 \mathrm{~nm}$ width at several distances from the interfacial plane.

The angles chosen to describe the water molecule are those proposed by Jedlovszky et $a l^{22}:$ i) the angle between the interface normal and the water dipole vector $(\theta)$; ii) the angle between the vector normal to the molecular plane and the projection of the interface normal onto the plane perpendicular to the dipole vector $(\varphi)$. Since one cannot distinguish between molecular normal vectors pointing in opposite directions, possible values of the angle $\varphi$ fall in the range $0^{\circ} \leq \varphi \leq 90^{\circ}$. This choice of vectors implies that an isotropic orientation of water molecules leads to uniform distributions of $\varphi$ and of $\cos (\theta)$. A more detailed description of this procedure, together with a diagram depicting the chosen angles and vectors was presented in the original publication by Jedlosvsky et al. $^{22}$.

The above method was applied to describe the orientation of the organic molecules as well. In this case, the chosen angles were: i) the angle between the interface normal and the vector bisecting the nitro group $(\theta)$; ii) the angle between the vector normal to the nitro group and the projection of the interface normal onto the plane perpendicular to the bisector vector $(\varphi)$. This is analogous to applying the same definition used for water molecules to the nitro group of NB and NPOE. We have concentrated on the orientation of the nitro group because this group is the one involved in hydrogen bonds with water ${ }^{8}$. Furthermore, even though the full nitrobenzene orientation cannot strictly be defined by only two angles, the orientation of the nitro group provides a good estimate of the orientation of the entire NB molecule, since it is preferentially aligned with the aromatic plane ${ }^{7}$. Unfortunately, this is not the case for NPOE, since the orientation of this molecule possesses many more degrees of freedom. Indeed, there is no simple 
way to uniquely define the orientation of the entire molecule, and thus we restrict ourselves to analyzing the orientation of the nitro group.

\subsection{Self-diffusion Coefficients}

The dynamic behavior of the interface was studied by calculating global and intrinsic profiles for the diffusion coefficients of water and organic molecules. The profiles were obtained using the procedure described in section 2.3 , but with $0.25 \mathrm{~nm}$ slices perpendicular to the $z$ direction. These slices are significantly thinner than those used in most previous studies, but the long simulation times used here allow for statistically meaningful results to be obtained. At the start of each sampling block, the reference time was set to zero and the positions of all sites were stored. In subsequent time steps, the squared displacement of each site relative to the reference position was computed. The displacement was assigned to the slice in which the molecule was located at the current time step. At the end of the sampling block, the mean-square displacement in each slice was calculated and the process was repeated until the end of the run. The diffusion coefficient in direction $x$, say, may then be obtained from a fit to the linear portion of the meansquare displacement plotted as a function of time, following Einstein's relation:

$$
\lim _{t \rightarrow \infty}\left\langle\left\|x_{i}(t)-x_{i}(0)\right\|^{2}\right\rangle=2 D_{x} t
$$

where $D_{\mathrm{x}}$ is the self-diffusion coefficient in the $x$ direction, $t$ is time and $x_{\mathrm{i}}(t)$ is the $x$ coordinate of particle $i$ at time $t$. Due to the geometry of the simulation box, diffusion in the $x$ and $y$ directions is indistinguishable. As such, our results are discussed in terms of the average value of these two coefficients, denoted $D_{\mathrm{xy}}$, and the value corresponding to diffusion in the direction perpendicular to the interface, $D_{\mathrm{z}}$.

\section{Results and Discussion}

\subsection{The Interface}


The procedure described in section 2.2 yields a system with the organic phase in the center of the simulation box, separated from the water phase by two interfaces. This is exemplified in Figure 2, where we show a snapshot obtained after equilibrating the water/NPOE interface. By visual inspection of the snapshot, it can be observed that there is very little extent of mixing between the two phases. Nevertheless, the interface is not flat but seems to exhibit strong corrugations.

\section{Figure 2}

A more detailed picture of the interface can be obtained by examining the probability distributions of interfacial width and position as we refine the resolution of the interfacial plane, by increasing the value of the parameter $N$ (see section 2.3). Results of such calculations for water/NPOE are shown in Figure 3. These results manifest the signature of a molecularly sharp interface that is broadened by thermal fluctuations. As we refine the resolution, we are able to capture more accurately the instantaneous position of the interfacial plane (the location halfway between the limits of each phase). The distribution in Figure 3 a becomes significantly broader as we increase $N$, and at the molecular level (largest $N$ shown) the position of the interfacial plane fluctuates widely. This shows clearly that the interfacial plane itself is significantly corrugated. The interfacial width distribution (Figure 3b), on the other hand, maintains approximately the same width, but its center shifts towards progressively lower values as $N$ increases. In other words, the limits of each phase are moving closer to each other as the resolution is refined. This means that the interface is relatively sharp on the molecular level. The picture of the water/NPOE interface arising from our simulation results is qualitatively similar to the water/NB interface ${ }^{14,30}$, as well as to other water/organic interfaces ${ }^{9,10,13,15,17-21}$.

\section{Figure 3}

Once we have established that the interface is sharp and corrugated, it is possible to compute intrinsic interfacial properties by applying the procedure described in section 2.3 , which 
eliminates fluctuations of the interfacial plane from the averaging process. In Figure 4, we show the result of this procedure applied to the calculation of the density profile for water/NPOE. This intrinsic density profile (thick line) is compared to the corresponding global profile (thin line), obtained with the usual averaging procedure based on a fixed reference frame, as well as to the intrinsic water/NB profile in a simulation box with the same cross-section. It is immediately clear that the intrinsic profiles of both water and organic show strong density oscillations in the interfacial region. The global profiles, on the contrary, are much smoother - a small first peak is still visible in the NPOE profile, but is practically absent in the case of water. This smoothing of the density profile results from corrugations of the interfacial plane brought about by thermal fluctuations. Only by decoupling these from fluctuations perpendicular to the interface are we able to obtain the true intrinsic density profiles for the liquid/liquid interface. Indeed, we have shown $^{30}$ that our water intrinsic density profiles were identical to those obtained by Chowdhary and Ladanyi ${ }^{29}$ using a different procedure.

\section{Figure 4}

In the intrinsic profiles for both water and organic, the density increases smoothly but steeply as we move from the opposite phase to the bulk region, until it reaches a first peak. The fact that the density rise is smooth, rather than a discontinuous step, suggests that the interface possesses some finite intrinsic width, in agreement with Weeks' interpretation of $\mathrm{CWT}^{42}$. The existence of a finite intrinsic width for liquid/liquid interfaces has been argued previously ${ }^{29,43,45}$, and supports the procedure outlined in section 2.4 for calculation of the interfacial tension. Following this method, we have fitted the global and intrinsic density profiles obtained in our simulations to equation (8). From the fits, the variances of the profiles were computed, using equation (9), and then substituted in equation (7) to estimate the capillary wave contribution to the total interfacial width. Finally, the interfacial tension for water/NPOE was calculated from 
equation (10) using data for two different values of $L$. The results of this procedure are shown in Table 1, together with values calculated previously ${ }^{30}$ for water/NB.

\section{Table 1}

In our previous publication ${ }^{30}$, we have shown that the procedure described above was able to yield interfacial tension values based on CWT that were consistent with direct calculations via the virial route - equation (5). The present results for the water/NPOE interface reinforce that claim. Indeed, the values of $\gamma$ obtained from the two methods are in good agreement with each other. The simulated interfacial tension for water/NPOE is higher than for water/NB. This is to be expected, because NPOE is not as polar as NB. The former can be thought of as a combination of a nitrobenzene molecule and an octane chain (see Figure 1). This aliphatic chain renders the molecule more hydrophobic, and thus increases its tension at the interface with water. Another conclusion that can be drawn from Table 1 is that the intrinsic width of the water/NPOE interface is smaller than for water/NB, which is probably also related to the increased hydrophobicity of NPOE. The intrinsic width reflects the extent of molecular-level mixing between the two phases, and this should be smaller for a more hydrophobic organic liquid. Finally, it is also clear that the bulk correlation length is larger for NPOE than for NB. If one considers that $\xi$ is closely related to the size of the organic molecule, a higher value for the bulkier NPOE is to be expected, and this is indeed the case. It should be noted, however, that the value of the bulk correlation length is somewhat sensitive to the details of the calculation procedure ${ }^{30}$.

The interfacial tension of NB calculated from the simulation with the OPLS potential is significantly higher than the experimental value ${ }^{48}$. A better estimate ${ }^{30}$ is provided by the model of Michael and Benjamin ${ }^{14}$, which was purposefully developed for nitrobenzene. Unfortunately, we have not been able to find experimental values for the water/NPOE interfacial tension in the available literature. Such values would provide a good test for the ability of the OPLS potential model to describe the interactions between water and NPOE. However, we have obtained a crude 
estimate of $\gamma$ for water/NPOE based on experimental data for the standard Gibbs energy of transfer of large ions across this interface ${ }^{49}$. Samec et al. ${ }^{49}$ argue that the Gibbs energy of transfer $(\Delta G)$ of large ions will depend mostly on a term that is related to solvophobic interactions. This term may be approximated using a semi-empirical expression that is proportional to the interfacial tension ${ }^{49,50}$. Thus, we have used their value of $\Delta G$ for the largest ion studied (the tetrapentylammonium cation) and applied equations (11-13) of their paper to extract a value for the interfacial tension of water/NPOE. This "experimental" value, shown also in Table 1, lies significantly below the simulation values. This is reasonable, given that we should expect the OPLS model to overestimate $\gamma$ for water/NPOE in the same way as for water/NB. Indeed, it is reassuring that the difference between the interfacial tension of water/NPOE and water/NB in the simulation $(3.3 \mathrm{mN} / \mathrm{m}$ if we use the CWT values) is similar to the difference between the corresponding experimental values $(3.5 \mathrm{mN} / \mathrm{m})$. Therefore, even though the OPLS model cannot provide accurate quantitative results for the interfacial tension of these interfaces, it does describe the trend of increasing $\gamma$ from NB to NPOE, and thus qualitatively captures the physics of the system. Agreement between simulation and experiment could probably be improved by tuning the unlike-pair interactions between water and organic molecules, but this is beyond the scope of this work.

\subsection{The Water Phase}

The presence of an interface induces changes in the organization of both liquids in its vicinity. In this section, we make use of intrinsic profiles calculated for several properties to shed light on the structure and dynamics of the water phase in the interfacial region. A closer examination of the intrinsic water density profile in Figure 4 shows the presence of a prominent peak adjacent to the interface. Beyond this peak, the density oscillations are progressively dampened until the bulk phase is reached. The first peak reveals a strong density enhancement in the liquid layer in immediate contact with the organic phase. As we will see later, water 
molecules in this first layer exhibit properties that are significantly different from bulk water. One striking conclusion that can be drawn from Figure 4 is that the water intrinsic profiles for the interfaces with NB and NPOE are extremely similar. This suggests that the structure of the water phase is not strongly affected by the nature of the opposite hydrophobic phase. Our subsequent analysis of other properties of water confirms this observation.

More detail about the structure of the water phase can be obtained by examining radial distribution functions calculated in slices parallel to the interfacial plane. Figure 5a shows RDFs between water oxygens $\left(\mathrm{O}_{\mathrm{W}}\right)$ based on a global definition of the interface, while Figure 5 b shows the same RDFs but based on a locally defined interfacial plane. The curves that are furthest away from the interface are identical to those obtained in pure water simulations ${ }^{31}$. As the interface is approached, density depletions in the surrounding environment cause a progressive decrease in the limiting value of the RDF and in the second peak. However, the first peak, characteristic of hydrogen bonding, remains practically intact even very close to the organic phase. This is reinforced in the intrinsic RDFs, which show that even water molecules that are protruding far into NPOE (last slice in Figure 5b) maintain their H-bonding character. This observation points to a very resilient hydrogen-bonded network for the water phase, persisting even in close vicinity to the organic liquid. Other water-water RDFs (not shown) corroborate these observations.

\section{Figure 5}

A more quantitative measure of the hydrogen bonding network of water is depicted in Figure 6. Here we show profiles for the number of water-water H-bonds per water molecule (thin dashed line), the number of neighbors in the first coordination shell (thick dashed line), the ratio between these two quantities (dotted line) and the number of water-NPOE bonds per water molecule (thin line). The latter are formed between water hydrogens $\left(\mathrm{H}_{\mathrm{W}}\right)$ and oxygen atoms belonging to the nitro group $\left(\mathrm{O}_{\mathrm{N}}\right)$, as shown in our previous simulation study of water dissolved in NPOE and $\mathrm{NB}^{8}$. In the center of the water phase, the number of neighbors and number of $\mathrm{H}$ - 
bonds per molecule exhibit values that are characteristic of bulk water. Moving toward the organic phase causes a reduction in the number of water-water hydrogen bonds, but also a decrease in the total number of neighbors. The ratio of these two values, however, increases as the interface is approached, which means that each interfacial water molecule is forming more hydrogen bonds per neighbor. Simultaneously, an increase in the number of bonds formed with NPOE is also evident close to the interface. These observations can be explained by an attempt on the part of water molecules to maximize the number of $\mathrm{H}$-bonds, thus maintaining their network as intact as possible even in the vicinity of the hydrophobic interface.

\section{Figure 6}

The intrinsic H-bond profiles show the same trend as the global ones, but the former can be related to the intrinsic density profiles. Thus, we can see that changes in the degree of hydrogen bonding only start to become evident close to the first density peak; water molecules that form the second peak (and beyond) show bulk values. The density enhancement in the water layer adjacent to the interface contributes to maintain the integrity of the hydrogen-bonded network, compensating for the presence of the organic phase. Molecules in this interfacial layer form very few bonds with nitro-group oxygens, and these only start to become significant for water molecules that protrude into the organic phase. As we move into this phase, the statistics worsen, due to the reduced number of water molecules. Nevertheless, we can clearly identify an increase in strength of the water-water bonds as well as an increase in the number of water-NPOE bonds. The trends shown in Figure 6 are very similar to the behavior observed in the water/NB system $^{30}$. The only minor difference is a somewhat higher value of water-NB bonds (relative to water-NPOE) for molecules deep within the organic phase, which can be attributed to the higher polarity of nitrobenzene.

The bivariate orientation profiles for water molecules, shown in Figures 7 and 8, further elucidate the effect of the interface on the structure of the water phase. To construct these figures, 
we have calculated the probability of finding a molecule with a given pair of angles $\theta$ and $\varphi$ in slices parallel to the interfacial plane. For each slice, areas colored in red correspond to higher angle-pair probabilities and blue areas correspond to lower probabilities. As expected based on the results discussed above, water molecules in the second density peak and in the bulk region adopt an isotropic orientation, typical of pure water (see last three slices in Figure 7 and last two slices in Figure 8). Molecules that form the first density peak, on the other hand, have a strong preference to arrange themselves with their molecular plane parallel to the interface $(\cos \theta=0$; $\varphi=0^{\circ}$ ). This allows them to maximize the number of hydrogen bonds with existing neighbors, in tune with the results presented in Figure 6. Thus, it is clear that the interfacial water layer, in immediate contact with the organic phase, forms a protective "wall" in which the molecules are closely packed and arranged so as to maximize the degree of water-water hydrogen bonding.

\section{Figure 7}

Due to the corrugations of the interface, described above, some water "fingers" extend into the core of the organic phase. The edges of these fingers are composed of molecules that adopt a distinct preferential orientation: with their molecular plane perpendicular to the interface and with one hydrogen atom almost parallel to the interface normal $\left(\cos \theta \approx 0.5 ; \varphi=90^{\circ}\right)$. This peak is evident in the global orientation profile (first two slices in Figure 7), but is reduced to a ridge in the intrinsic profile. This confirms that we are not dealing with a layer of water molecules that are perpendicularly oriented, but rather with a restricted number of molecules those that are almost completely surrounded by organic molecules. Their preferred orientation allows for the formation of a hydrogen bond with another water molecule in the parallel layer, as pointed out by Jedlovszky et $a .^{22}$, but also allows for the formation of a bond with the nitro group of NPOE, via the protruding hydrogen atom. In our previous work ${ }^{30}$, we have observed a very similar behavior at the water/NB interface and have presented a diagram illustrating these preferred orientations (see Figure 15 of Ref. 30), which have a pronounced effect on the 
orientation of interfacial nitrobenzene molecules. As we will see below, this effect is also present for NPOE, but it is less pronounced.

\section{Figure 8}

We complete our analysis of the water phase by looking at the profiles for the selfdiffusion coefficient, shown in Figure 9. The diffusion parallel to the interface in the bulk region tends toward the value calculated independently for pure water ${ }^{8}$. However, this coefficient decreases slightly as we move toward the NPOE phase. NPOE molecules are much slower than water, and thus their presence induces a decrease in mobility of the latter. The decrease in the water diffusion coefficient is more pronounced for NPOE than for $\mathrm{NB}^{30}$, which can be explained by the much lower bulk diffusion coefficient of NPOE (by more than an order of magnitude) ${ }^{7}$.

\section{Figure 9}

A more interesting trend is observed in the diffusion perpendicular to the interface: the curve exhibits a minimum close to the interface. The decrease in perpendicular mobility of the interfacial water molecules is most likely due to the nature of the protective "wall" described above. In this interfacial layer, the density is higher, molecules are more closely packed and form stronger hydrogen bonds. Thus, it is reasonable to expect reduced motion in the direction normal to the interfacial plane (which would otherwise disrupt the tightly packed layer). As we move further into the organic phase, this effect dies out and the perpendicular diffusion coefficient increases again. This minimum in $D_{\mathrm{z}}$ has also been observed in our current and previous ${ }^{30}$ simulations of the water/NB interface. Another aspect that is worth noticing is that the interface has a much stronger effect on $D_{\mathrm{z}}$ than on $D_{\mathrm{xy}}$. Indeed, the former does not even reach bulk values in the center of the water phase, which suggests that the simulation box is not large enough to eliminate all effects of the interface (as seen above, the effect is not so pronounced for thermodynamic and structural properties, and bulk values are attained).

\subsection{The Organic Phase}


The intrinsic density profiles for the organic liquids (see Figure 4) exhibit oscillations that persist much further into the bulk region than in the case of water. Furthermore, the NB profile is significantly different from that of NPOE. The first major difference is the higher density reached in the bulk phase by NB. The bulk density values of both organic liquids agree well with independent calculations from pure-component simulations ${ }^{7}$. The other main difference is that the density oscillations persist for longer distances in the case of NPOE, relative to NB. This can be explained by the fact that the NPOE molecule is much larger and bulkier than the NB molecule, and thus more sensitive to packing constraints ${ }^{7}$ imposed by the interface.

\section{Figure 10}

In Figure 10, we show the RDFs between carbon atoms attached to the nitro group $\left(\mathrm{C}_{\mathrm{N}}\right)$ of NB at several distances to the interface. Far from the interface, the curves are identical to those obtained in simulations of the pure component ${ }^{7}$. The depletion in density caused by the presence of the water phase brings about a decrease in the limiting value of the RDF and in the intensity of the main peak. However, one can notice a change of shape in the RDF as we approach the water phase. The features at shorter distances (shoulder at $0.4 \mathrm{~nm}$ and first part of the main peak, at $0.55 \mathrm{~nm}$ ) become more prominent in the region just prior to the interfacial layer (e.g., second and third slices in Figure 10b). The strengthening of these features is a reflection of NB molecules with an antiparallel alignment ${ }^{51}$.

\section{Figure 11}

\section{Figure 12}

More information regarding the molecular organization of nitrobenzene at the interface can be obtained from the bivariate orientation profiles (see Figures 11 and 12). From these plots, we can see that bulk molecules, as expected, adopt an isotropic orientation. As we move closer to the interface, we can distinguish a preference for orientation perpendicular to the interfacial plane $(\cos \theta=-1$ or 1$)$. Molecules in this region, which are not in direct contact with water, are aligned 
in an antiparallel fashion and are responsible for the enhancement of the short-distance features in the RDFs of Figure 10. Following this layer, we observe a peak at $\cos \theta=0$ and $\varphi=90^{\circ}$, characteristic of molecules that are oriented with both their dipole and their normal vectors parallel to the interfacial plane. This orientation allows for the formation of a hydrogen bond between the oxygen atom of $\mathrm{NB}$, which points to the water phase, and the hydrogen atom of water, which is protruding into the organic phase (see Figure 7). Finally, we encounter the tightly-packed interfacial layer (corresponding to the main peak in the density profile of Figure 4), where the NB molecules are oriented with their molecular planes parallel to the interface (cos $\theta=0$ and $\varphi=0^{\circ}$ ). This detailed orientation profile is qualitatively similar to that obtained previously using a different model for $\mathrm{NB}^{30}$.

\section{Figure 13}

We move now to an analysis of the structure of interfacial NPOE, starting with the $\mathrm{C}_{\mathrm{N}}-\mathrm{C}_{\mathrm{N}}$ RDFs shown in Figure 13. Once again, the curves tend toward the pure component results ${ }^{7}$ in the bulk region. The NPOE molecule is more complex than NB, due to the presence of the alkyl chain, and thus it becomes more difficult to draw conclusions from the RDFs. Nevertheless, a region can be identified, particularly in the intrinsic profile of Figure $13 \mathrm{~b}$, where the shoulder at $0.45 \mathrm{~nm}$ becomes more prominent. In analogy with nitrobenzene, this peak is probably due to molecules packed with their aromatic rings in an antiparallel arrangement. This type of arrangement can also be inferred from the bivariate orientation profiles, shown in Figures 14 and 15. From these plots, it appears that the tendency for antiparallel alignment is even more pronounced than for NB. Indeed, we can identify three distinct regions (as opposed to only two for NB) where the NPOE nitro groups are oriented perpendicularly to the interfacial plane with their bisector vector pointing alternately to the water phase $(\cos \theta=1)$ and away from the water phase $(\cos \theta=-1)$. This arrangement is present even in the bulk region (e.g., last slice of Figure 
15), which shows that structural anisotropies imposed by the interface are much more persistent in NPOE than in NB.

\section{Figure 14}

\section{Figure 15}

As we move closer to the interface, the preference for perpendicular orientation gradually shifts to a parallel arrangement $\left(\cos \theta=0\right.$ and $\left.\varphi=0^{\circ}\right)$, and this is the preferred orientation in the interfacial layer (corresponding to the main peak in the density profile). The peak at $\cos \theta=0$ and $\varphi=90^{\circ}$ observed for NB is reduced to a small ridge in the case of NPOE (e.g., third slice in Figure 14). Considering that this orientation, in which an oxygen atom points into the water phase, is the main responsible for water-organic hydrogen bonds, one can relate the less prominent peak to the reduced number of such bonds relative to NB (see Figure 6). This may be caused by steric interference from the alkyl chains, which does not favor this type of orientation. In general, one can say that interfacial NPOE molecules are less organized than NB, and this is most likely due to the presence of the hydrophobic chain in the former.

Finally, we look at the NB and NPOE diffusion coefficients, shown in Figures 16 and 17, respectively. The NB profiles for lateral diffusion show a small increase in the diffusion coefficient from bulk values as the interface is crossed. This effect of increased mobility caused by the contact with faster water molecules is analogous to that observed for water (Figure 9), but in the opposite direction. Also in analogy with water, the perpendicular diffusion coefficient of NB molecules shows a minimum in the vicinity of the interface. This minimum is caused by the reduced mobility in the close-packed interfacial layer. The two profiles converge as we move further into the bulk of the water phase.

\section{Figure 16}

The lateral diffusion profiles for NPOE (open symbols in Figure 17) show a similar trend to the NB results, except that the increase in mobility caused by the contact with water molecules 
is much more substantial (up to a fourfold increase in $D_{\mathrm{xy}}$ ). NPOE molecules in the bulk region experience significant entanglement of the alkyl chains, manifested by close contacts present in RDFs between NPOE chain atoms ${ }^{7}$. This entanglement is the main cause for the observed strong reduction of the pure-component diffusion coefficient (and concomitant increase in viscosity) of $\mathrm{NPOE}$ relative to $\mathrm{NB}^{7}$. As we approach the interface, contact with smaller and more mobile water molecules reduces this entanglement, increasing the diffusion coefficient. The influence of water is also felt in the case of $\mathrm{NB}$, but less so, since nitrobenzene does not suffer from chain entanglement.

\section{Figure 17}

$D_{\mathrm{z}}$, however, shows a remarkably different trend compared to NB: it increases monotonically and does not exhibit a minimum at the interface. This is despite the fact that a tightly packed layer of NPOE molecules also exists at the interface with water (see Figure 4). The observed trend is the result of two competing effects. On the one hand, the reduction of the chain entanglement caused by the contact with water (as discussed above) brings about a very strong increase in mobility. On the other hand, the effect of the interfacial layer is to reduce the degree of perpendicular mobility. In water and NB, which are more mobile, the latter effect is more significant than the former, and a minimum appears in the profiles for $D_{\mathrm{z}}$. In NPOE, however, the first effect is much stronger, and the minimum is reduced to a region of constant $D_{\mathrm{z}}$. In other words, the increase in mobility caused by the presence of water is sufficient to offset the effect of the density increase in the interfacial layer.

\section{Conclusions}

In this paper, we have presented for the first time simulation results of the interface between water and 2-nitrophenyloctyl ether. This organic solvent is very important in experimental electrochemical studies, and is seen as a promising alternative to traditional liquids 
such as nitrobenzene. Our results show that the water/NPOE interface is molecularly sharp, but corrugated by capillary waves. These waves are suppressed in NPOE relative to NB, due to the higher degree of hydrophobicity of the former, causing an increase in the value of the interfacial tension. Values of this property calculated using capillary wave theory agree with estimates from the virial route, further validating the methodology proposed in our previous work ${ }^{30}$.

Our method of decoupling fluctuations of the interfacial plane from the averaging procedure allows for the calculation of intrinsic properties of the system. These provide a detailed picture of the molecular organization of the water and organic phases in the vicinity of the interface. In both liquids, our results reveal the presence of a tightly-packed interfacial layer, responsible for a density enhancement at the interface and composed of molecules oriented with their planes parallel to the interface. Water molecules organize themselves so as to maximize the extent of hydrogen bonding, be it with other water molecules or with the nitro groups of the organic molecules. The extent of water-organic bonds is reduced in NPOE, relative to NB, once more due to the increased hydrophobic character of the former. Despite the differences between the two solvents, the structure of the water phase is practically identical in both interfaces, suggesting that it does not depend strongly on the nature of the hydrophobic phase. This is in agreement with previous comparisons of different aqueous interfaces ${ }^{24,29}$.

The major difference between NPOE and NB is the presence of a long alkyl chain in the former. This imposes steric constraints that are responsible for the observed differences in molecular organization. Interfacial NPOE molecules tend to be less organized than NB molecules. In particular, the interfacial layer is not as clear-cut as in the case of nitrobenzene, and the preferred orientation that leads to water-organic hydrogen bonds is not as prominent. However, anisotropies imposed by the interface persist much further into the bulk region than for $\mathrm{NB}$, which is due to the bulkier nature of the NPOE molecule. Entanglement between the alkyl chains in the bulk region causes a significant decrease in the diffusion coefficient of NPOE (more 
than an order of magnitude lower than for NB). As the interface is approached, however, the contact with smaller and more mobile water molecules reduces this effect and increases the diffusion coefficient. This effect dominates the dynamic behavior of interfacial NPOE molecules and explains the differences observed relative to NB (where the entanglement effect is absent). It is likely that these differences in interfacial dynamic behavior will have a pronounced effect on the transfer of solutes across the interface with water ${ }^{49}$. Molecular dynamics simulations of ion transfer across the water/NB and water/NPOE interfaces are underway in our laboratory to shed light on this issue.

\section{Acknowledgments:}

Thanks are due to Fundação para a Ciência e Tecnologia (FCT), Lisbon, Portugal and to FEDER for financial support to REQUIMTE.

\section{References:}

[1] Volkov, A. G. Liquid Interfaces in Chemical, Biological and Pharmaceutical Applications; Marcel-Dekker: New York, 2001; Vol. 95, Chapter 2.

[2] Benjamin, I. Annu. Rev. Phys. Chem. 1997, 48, 407.

[3] Marcus, Y. Ion Properties; Marcel-Dekker: New York, 1997.

[4] Samec, Z.; Langmaier, J.; Trojanek, A. J. Electroanal. Chem. 1996, 409, 1.

[5] Scholz, F.; Gulaboski, R. ChemPhysChem 2005, 6, 16.

[6] Gulaboski, R.; Galland, A.; Bouchard, G.; Caban, K.; Kretschmer, A.; Carrupt, P. -A.; Girault, H. H.; Scholz, F. J. Phys. Chem. B. 2004, 108, 4565.

[7] Jorge, M.; Gulaboski, R.; Pereira, C. M.; Cordeiro, M. N. D. S. J. Phys. Chem. B 2006, 110, 12530.

[8] Jorge, M.; Gulaboski, R.; Pereira, C. M.; Cordeiro M. N. D. S. Mol. Phys. 2006, 104, 3627. 
[9] Linse, P. J. Chem. Phys. 1987, 86, 4177.

[10] Benjamin, I. J. Chem. Phys. 1992, 97, 1432.

[11] Buff, F.; Lovett, R.; Stillinger, F. Phys. Rev. Lett. 1965, 15, 621.

[12] Rowlinson, J; Widom, B. Molecular Theory of Capillarity; Clarendon Press: Oxford, 1982.

[13] Michael, D.; Benjamin, I. J. Phys. Chem. 1995, 99, 1530.

[14] Michael, D.; Benjamin, I. J. Electroanal. Chem. 1998, 450, 335.

[15] Fernandes, P. A.; Cordeiro, M. N. D. S.; Gomes, J. A. N. F. J. Phys. Chem. B 1999, 103, 6290.

[16] Fernandes, P. A.; Cordeiro, M. N. D. S.; Gomes, J. A. N. F. J. Phys. Chem. B 1999, 103, 8930.

[17] da Rocha, S. R. P.; Johnston, K. P.; Westacott, R. E.; Rossky, P. J. J. Phys. Chem. B 2001, $105,12092$.

[18] Cordeiro, M. N. D. S. Mol. Simul. 2003, 29, 817.

[19] Chang, T.-M.; Dang, L. X. J. Chem. Phys. 1996, 104, 6772.

[20] Zhang, Y.; Feller, S. E.; Brooks, B. R.; Pastor, R. W. J. Chem. Phys. 1995, 103, 10252.

[21] Patel, H. A.; Nauman, E. B.; Garde, S. J. Chem. Phys. 2003, 119, 9199.

[22] Jedlovszky, P.; Vincze, A.; Horvai, G. J. Chem. Phys. 2002, 117, 2271.

[23] Wang, H.; Carlson, E.; Henderson, D.; Rowley, R. L. Mol. Simul. 2003, 29, 777.

[24] Jedlovszky, P.; Vincze, A.; Horvai, G. J. Mol. Liq. 2004, 109, 99.

[25] Chacón, E.; Tarazona, P. Phys. Rev. Lett. 2003, 91, 166103.

[26] Tarazona, P.; Chacón, E. Phys. Rev. B 2004, 70, 235407.

[27] Chacón, E.; Tarazona, P. J. Phys.: Condens. Matter 2005, 17, S3493.

[28] Chacón, E.; Tarazona, P.; Alejandre, J. J. Chem. Phys. 2006, 125, 014709.

[29] Chowdhary, J.; Ladanyi, B. M. J. Phys. Chem. B 2006, 110, 15442. 
[30] Jorge, M.; Cordeiro, M. N. D. S. J. Phys. Chem. B 2007, 111, 17612.

[31] Berendsen, H. J. C.; Grigera, J. R.; Straatsma, T. P. J. Phys. Chem. 1997, 91, 6269.

[32] Jorgensen, W. L.; Tirado-Rives, J. J. Am. Chem. Soc. 1988, 110, 1657.

[33] Price, M. L. P.; Ostrovsky, D.; Jorgensen, W. L. J. Comput. Chem. 2001, 22, 1340.

[34] Essman, U.; Perela, L.; Berkowitz, M. L.; Darden, T.; Lee, H.; Pedersen, L. G. J. Chem. Phys. 1995, 103, 8577.

[35] Berendsen, H. J. C.; van der Spoel, D.; van Drunen, R. Comput. Phys. Commun. 1995, 91, 43.

[36] Lindahl, E.; Hess, B.; van der Spoel, D. J. Mol. Mod. 2001, 7, 306.

[37] Hockney, R. W.; Goel, S.P.J. J. Comput. Phys. 1974, 14, 148.

[38] Hess, B.; Bekker, H.; Berendsen, H. J. C.; Fraaije, J. G. E. M. J. Comput. Chem. 1997, 18, 1463.

[39] Nosé, S. Mol. Phys. 1984, 52, 255.

[40] Hoover, W. G. Phys. Rev. A 1985, 31, 1695.

[41] Parrinello, M.; Rahman, A. J. Appl. Phys. 1981, 52, 7182.

[42] Weeks, J. D. J. Chem. Phys. 1977, 67, 3106.

[43] Senapati, S.; Berkowitz, M. L. Phys. Rev. Lett. 2001, 87, 176101.

[44] Mitrinovic, D. M.; Tikhonov, A. M.; Li, M.; Huang, Z.; Schlossman, M. L. Phys. Rev. Lett. 2000, 85, 582.

[45] Sides, S. W.; Grest, G. S.; Lacasse, M.-D. Phys. Rev. E 1999, 60, 6708.

[46] Toxvaerd, S.; Stecki, J. J. Chem. Phys. 1995, 102, 7163.

[47] van Buuren, A. R.; Marrink, S.-J.; Berendsen, H. J. C. J. Phys. Chem. 1993, 97, 9206.

[48] Markin, V. S.; Volkov, A. G.; Volkova-Gugeshashvili, M. I. J. Phys. Chem. B 2005, 109, 16444.

[49] Samec, Z.; Langmaier, J.; Trojánek, A. J. Electroanal. Chem. 1996, 409, 1. 
[50] Markin, V. S.; Volkov, A.G. Electrochim. Acta 1989, 34, 93.

[51] Janssen, R. H. C.; Theodorou, D. N.; Raptis, S.; Papadopoulos, M.G. J. Chem. Phys. 1999, $111,9711$. 
Table 1 - Intrinsic interfacial widths, interfacial tensions and bulk correlation lengths for the studied interfaces.

\begin{tabular}{l|ll} 
& water/NPOE & water/NB \\
\hline$w_{\mathrm{i}}(\mathrm{nm})^{\mathrm{a}}$ & $0.0428 \pm 0.0011$ & $0.0487 \pm 0.0005$ \\
$\gamma_{\exp }(\mathrm{mN} / \mathrm{m})$ & $29^{\mathrm{c}}$ & $25.5^{\mathrm{d}}$ \\
$\gamma_{\mathrm{N}}(\mathrm{mN} / \mathrm{m})^{\mathrm{b}}$ & $45.5 \pm 3.8$ & $39.7 \pm 3.3$ \\
$\gamma_{\mathrm{cw}}(\mathrm{mN} / \mathrm{m})^{\mathrm{a}}$ & 43.2 & 39.9 \\
$\xi(\mathrm{nm})^{\mathrm{a}}$ & 0.99 & 0.58
\end{tabular}

a - Calculated from CWT with fits to the density profiles.

${ }^{b}$ - Calculated from the virial route.

c - Estimated from values of $\Delta G$ of ion transfer taken from Ref. 49.

d - Taken from Ref. 48. 
Figure 1. Schematic diagram depicting the molecular structure of NB (a) and NPOE (b), as well as the nomenclature used for each atom type.

Figure 2. Simulation snapshot of the water/NPOE interface with $L=3.5 \mathrm{~nm}$ showing the shape of the simulation box and the coordinate axes. Oxygen atoms are shown in blue, carbon atoms in purple, nitrogen atoms in green and hydrogen atoms in white.

Figure 3. Probability distributions of the interface position (a) and width (b) for the water/NPOE interface with $L=3.5 \mathrm{~nm}$ at different values of $N$ (number of subdivisions on each side of a square mesh parallel to the interfacial plane - see text). The distributions are averaged over both interfaces.

Figure 4. Density profiles for the water/organic interfaces with $L=3.5 \mathrm{~nm}$ : thin line - global water/NPOE profile; thick line - intrinsic water/NPOE profile; dashed line - intrinsic water/NB profile. The interface is at the origin, with water profiles on the left (negative values of $z$ ) and organic profiles on the right (positive values of $z$ ).

Figure 5. Global (a) and intrinsic (b) $\mathrm{O}_{\mathrm{W}}-\mathrm{O}_{\mathrm{W}}$ radial distribution functions for the system water/NPOE with $L=4.5 \mathrm{~nm}$ in slices perpendicular to the interface. The curves, from top to bottom, correspond to positions relative to the limit of the organic phase of: $-0.9,-0.7,-0.5,-0.3,-$ $0.1,0.1,0.3,0.5,0.7$ and $0.9 \mathrm{~nm}$ (positive values are within the organic phase). The last two slices of part b) are not shown because they are not statistically significant.

Figure 6. Global (a) and intrinsic (b) hydrogen bond profiles for the water/NPOE interface with $L=4.5 \mathrm{~nm}$ : thick dashed line - number of molecules in the first coordination shell; thin dashed line - number of water-water hydrogen bonds per molecule; full line - proportion of bonded water molecules; dotted line - number of water-NPOE hydrogen bonds per water molecule. The water density profiles (thick lines) are superimposed for ease of visualization. Negative values are within the water phase. 
Figure 7. Global orientation distribution of water molecules for the system water/NPOE with $L=3.5 \mathrm{~nm}$ calculated in slices perpendicular to the interface. Slices, from top to bottom, correspond to positions relative to the limit of the organic phase of: $0.9,0.7,0.5,0.3,0.1,-0.1,-$ 0.3, -0.5 and $-0.7 \mathrm{~nm}$ (negative values are within the water phase). Red corresponds to high normalized probability and blue to low probability.

Figure 8. Intrinsic orientation distribution of water molecules for the system water/NPOE with $L=3.5 \mathrm{~nm}$ calculated in slices perpendicular to the interface. Slices, from top to bottom, correspond to positions relative to the limit of the organic phase of: $0.3,0.1,-0.1,-0.3,-0.5$ and $0.7 \mathrm{~nm}$ (slices closer to the organic phase are not statistically meaningful). Red corresponds to high normalized probability and blue to low probability.

Figure 9. Water diffusion coefficient profiles, global (a) and intrinsic (b), for the water/NPOE interface with $L=4.5 \mathrm{~nm}$. Open symbols are for diffusion in the plane of the interface, closed symbols are for diffusion perpendicular to the interface and the dashed line is the purecomponent diffusion coefficient. The water density profiles (thick lines) are superimposed for ease of visualization. Negative values are within the water phase.

Figure 10. Global (a) and intrinsic (b) $\mathrm{C}_{\mathrm{N}}-\mathrm{C}_{\mathrm{N}} \mathrm{RDFs}$ for the system water/NB with $L=3.5 \mathrm{~nm}$ in slices perpendicular to the interface. The curves, from top to bottom, correspond to positions relative to the limit of the water phase of: $0.9,0.7,0.5,0.3,0.1,-0.1,-0.3$, and -0.5 (the last three curves of part b) are not statistically meaningful). The first curves are highlighted with thick lines for clarity.

Figure 11. Global orientation distributions of NB molecules for the system water/NB with $L=3.5$ nm calculated in slices perpendicular to the interface. Slices, from top to bottom, correspond to positions relative to the limit of the organic phase of: $-0.9,-0.7,-0.5,-0.3,-0.1,0.1,0.3,0.5$ and $0.7 \mathrm{~nm}$ (positive values are within the organic phase). Red corresponds to high normalized probability and blue to low probability. 
Figure 12. Intrinsic orientation distributions of $\mathrm{NB}$ molecules for the system water/NB with $L=3.5 \mathrm{~nm}$ calculated in slices perpendicular to the interface. Slices, from top to bottom, correspond to positions relative to the limit of the organic phase of: $-0.1,0.1,0.3,0.5,0.7$ and 0.9 (slices closer to the water phase are not statistically meaningful). Red corresponds to high normalized probability and blue to low probability.

Figure 13. Global (a) and intrinsic (b) $\mathrm{C}_{\mathrm{N}}-\mathrm{C}_{\mathrm{N}}$ RDFs for the system water/NPOE with $L=4.5 \mathrm{~nm}$ in slices perpendicular to the interface. Lines and distances to the limit of the water phase are the same as in Figure 10.

Figure 14. Global orientation distributions of NPOE molecules for the system water/NPOE with $L=3.5 \mathrm{~nm}$ calculated in slices perpendicular to the interface. Colors and distances to the limit of the water phase are the same as in Figure 12.

Figure 15. Intrinsic orientation distributions of NPOE molecules for the system water/NPOE with $L=3.5 \mathrm{~nm}$ calculated in slices perpendicular to the interface. Colors and distances to the limit of the water phase are the same as in Figure 13.

Figure 16. NB diffusion coefficient profiles, global (a) and intrinsic (b) for the system water/NB at $L=3.5 \mathrm{~nm}$. Open symbols are for diffusion in the plane of the interface, closed symbols are for diffusion perpendicular to the interface and the dashed line is the pure-component diffusion coefficient. The organic density profiles (thick lines) are superimposed for ease of visualization. Positive values are within the organic phase.

Figure 17. NPOE diffusion coefficient profiles, global (a) and intrinsic (b) for the system water/NPOE at $L=4.5 \mathrm{~nm}$. Lines are the same as in Figure 16. Positive values are within the organic phase. 

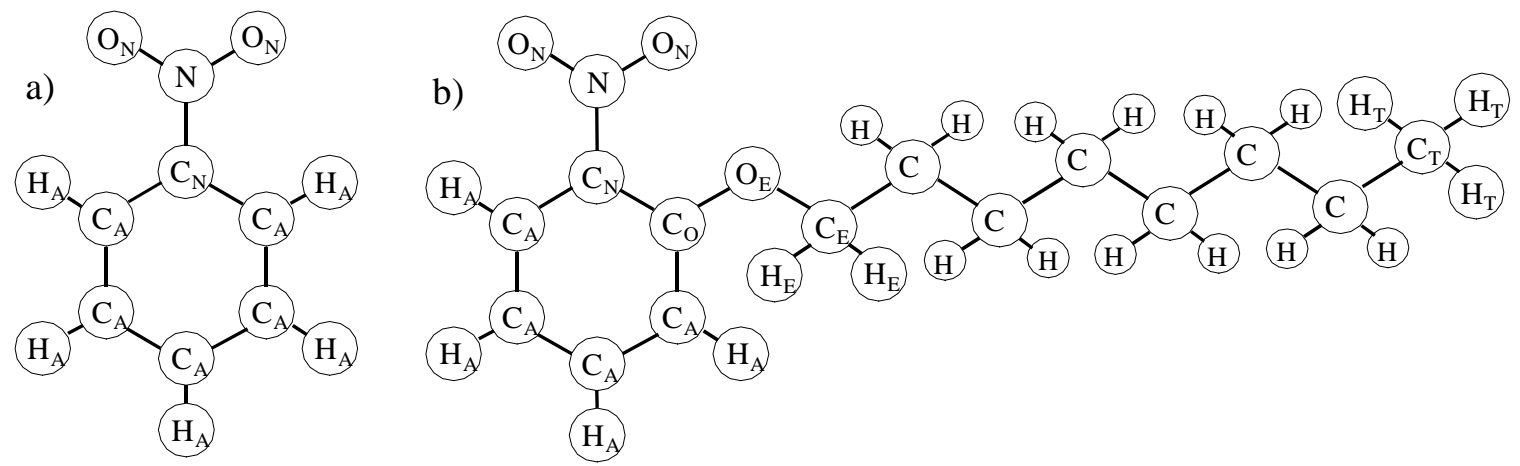

Figure 1. Schematic diagram depicting the molecular structure of NB (a) and NPOE (b), as well as the nomenclature used for each atom type. 


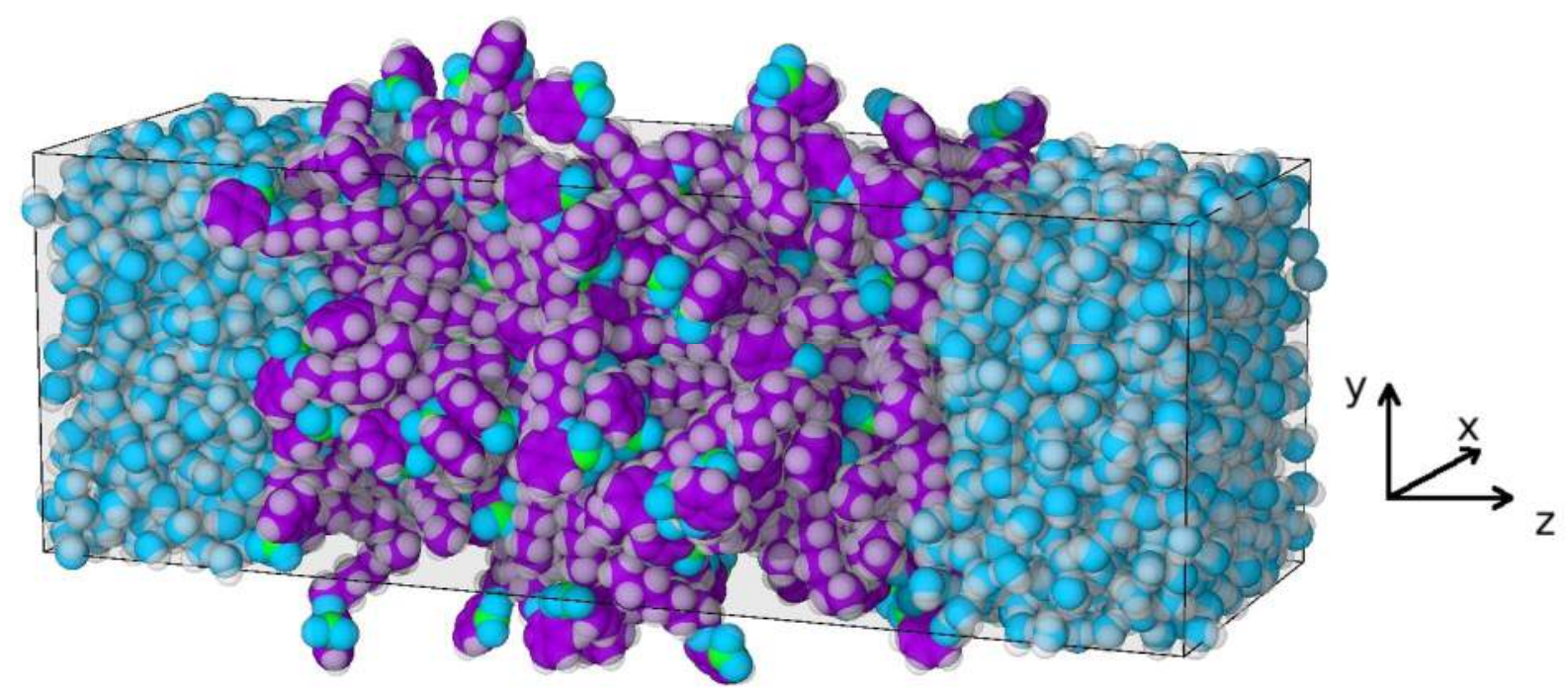

Figure 2. Simulation snapshot of the water/NPOE interface with $L=3.5 \mathrm{~nm}$ showing the shape of the simulation box and the coordinate axes. Oxygen atoms are shown in blue, carbon atoms in purple, nitrogen atoms in green and hydrogen atoms in white. 

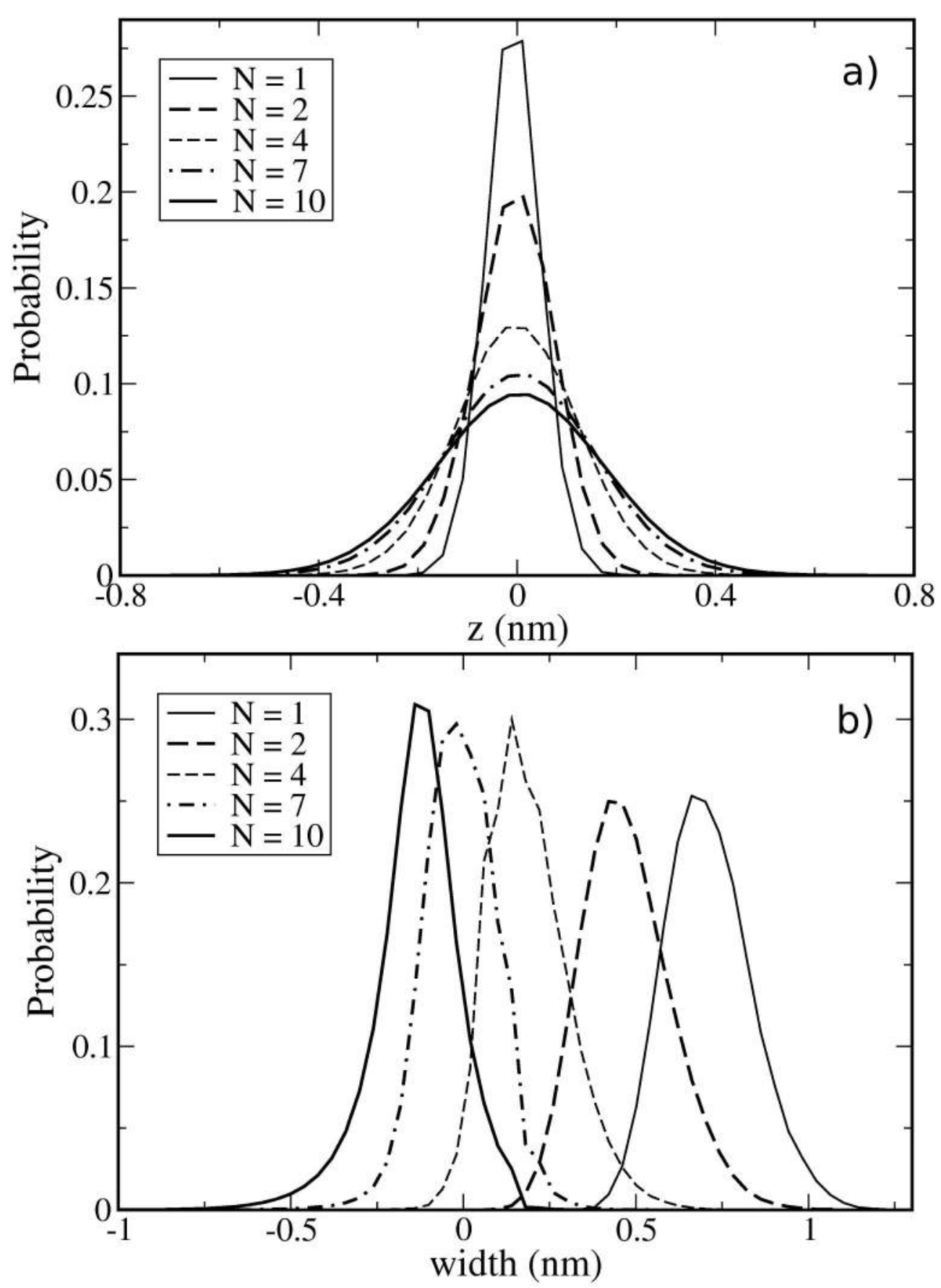

Figure 3. Probability distributions of the interface position (a) and width (b) for the water/NPOE interface with $L=3.5 \mathrm{~nm}$ at different values of $N$ (number of subdivisions on each side of a square mesh used to define the interfacial plane - see text). The distributions are averaged over both interfaces. 


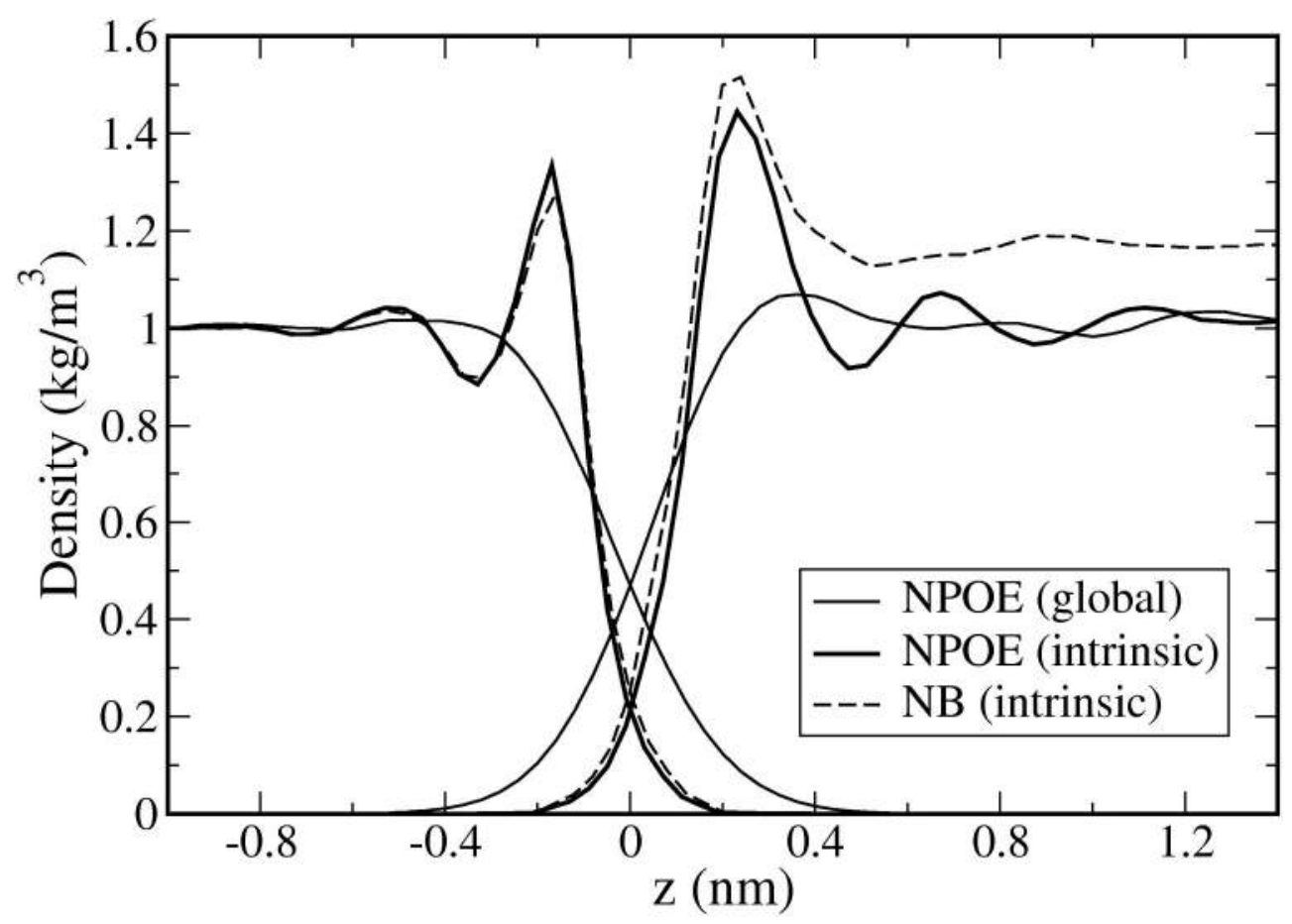

Figure 4. Density profiles for the water/organic interfaces with $L=3.5 \mathrm{~nm}$ : thin line - global water/NPOE profile; thick line - intrinsic water/NPOE profile; dashed line - intrinsic water/NB profile. The interface is at the origin, with water profiles on the left (negative values of $z$ ) and organic profiles on the right (positive values of $z$ ). 

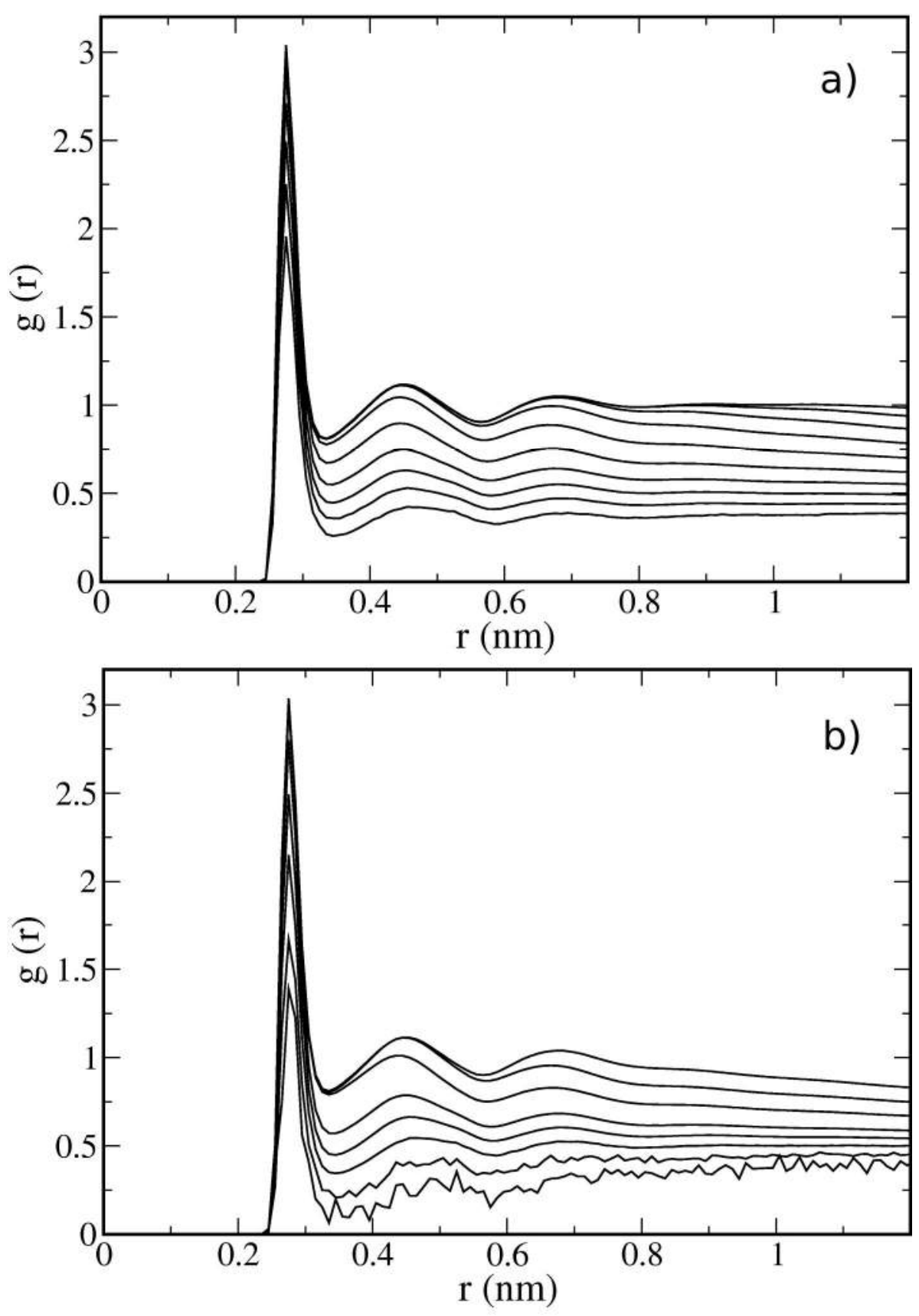

Figure 5. Global (a) and intrinsic (b) $\mathrm{O}_{\mathrm{W}}-\mathrm{O}_{\mathrm{W}}$ radial distribution functions for the system water/NPOE with $L=4.5 \mathrm{~nm}$ in slices perpendicular to the interface. The curves, from top to bottom, correspond to positions relative to the limit of the organic phase of: $-0.9,-0.7,-0.5,-0.3,-$ $0.1,0.1,0.3,0.5,0.7$ and $0.9 \mathrm{~nm}$ (positive values are within the organic phase). The last two slices of part b) are not shown because they are not statistically significant. 

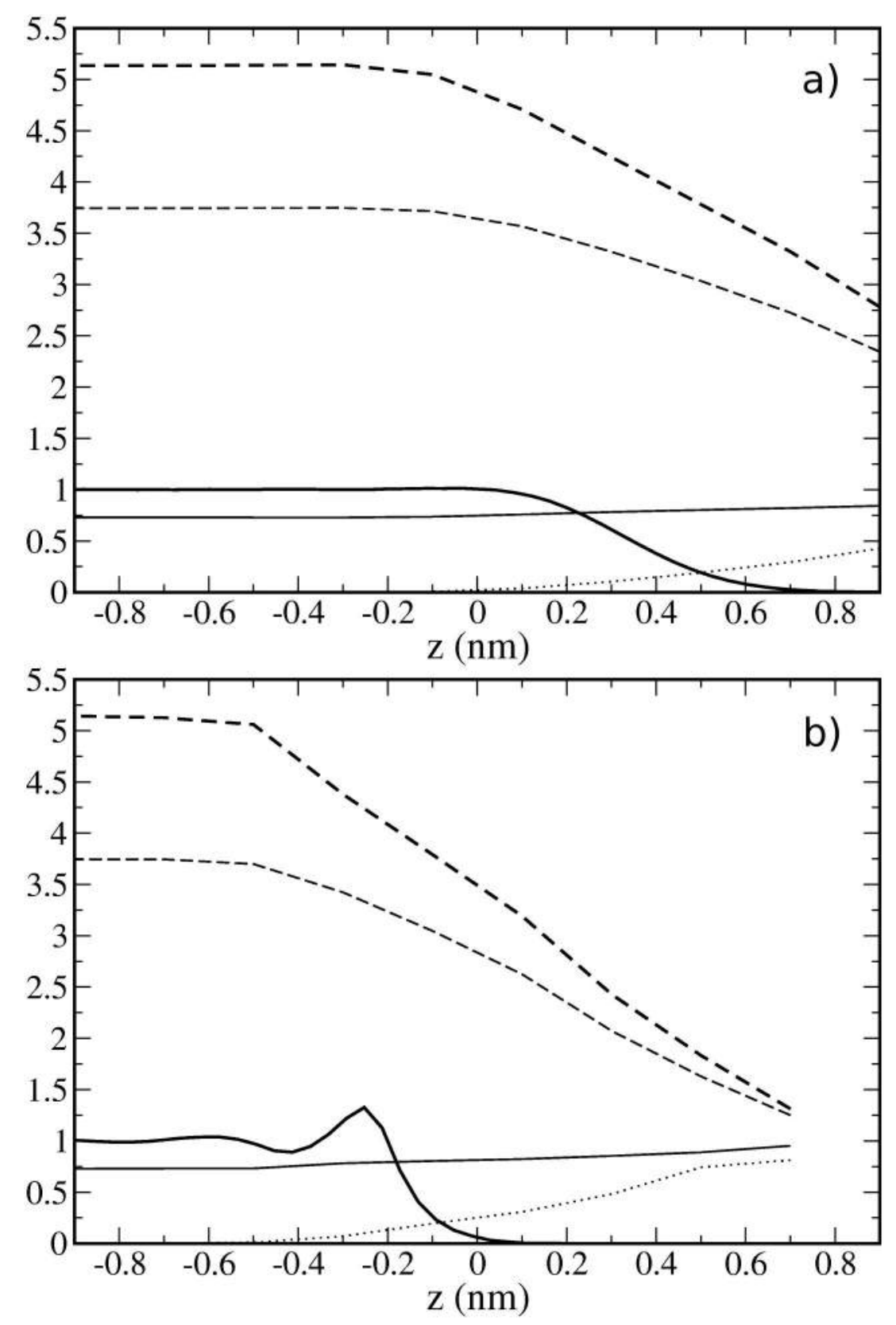

Figure 6. Global (a) and intrinsic (b) hydrogen bond profiles for the water/NPOE interface with $L=4.5 \mathrm{~nm}$ : thick dashed line - number of molecules in the first coordination shell; thin dashed line - number of water-water hydrogen bonds per molecule; full line - proportion of bonded water molecules; dotted line - number of water-NPOE hydrogen bonds per water molecule. The water density profiles (thick lines) are superimposed for ease of visualization. Negative values are within the water phase. 

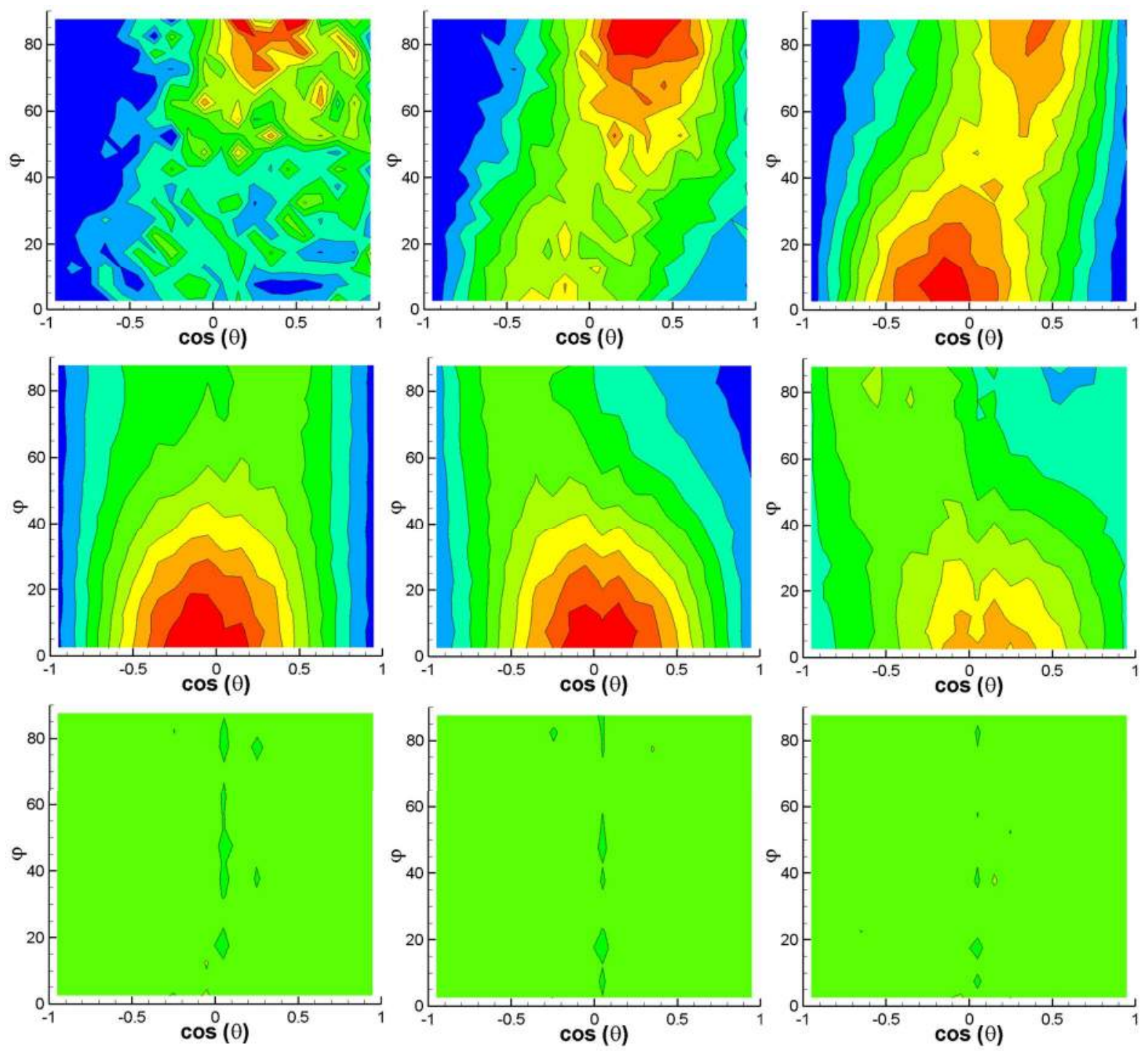

Figure 7. Global orientation distribution of water molecules for the system water/NPOE with $L=3.5 \mathrm{~nm}$ calculated in slices perpendicular to the interface. Slices, from top to bottom, correspond to positions relative to the limit of the organic phase of: $0.9,0.7,0.5,0.3,0.1,-0.1$, 0.3, -0.5 and $-0.7 \mathrm{~nm}$ (negative values are within the water phase). Red corresponds to high normalized probability and blue to low probability. 

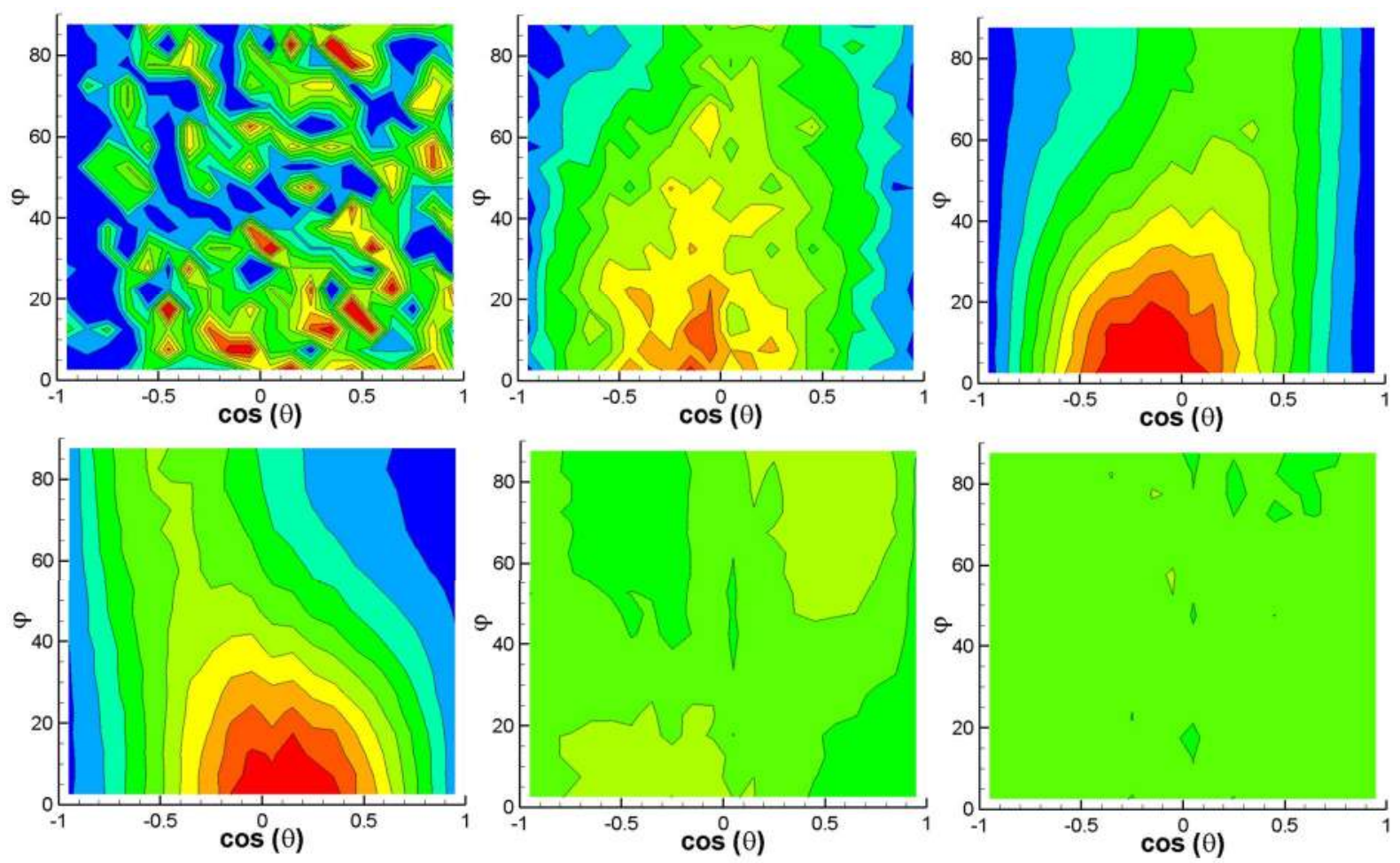

Figure 8. Intrinsic orientation distribution of water molecules for the system water/NPOE with $L=3.5 \mathrm{~nm}$ calculated in slices perpendicular to the interface. Slices, from top to bottom, correspond to positions relative to the limit of the organic phase of: $0.3,0.1,-0.1,-0.3,-0.5$ and $0.7 \mathrm{~nm}$ (slices closer to the organic phase are not statistically meaningful). Red corresponds to high normalized probability and blue to low probability. 

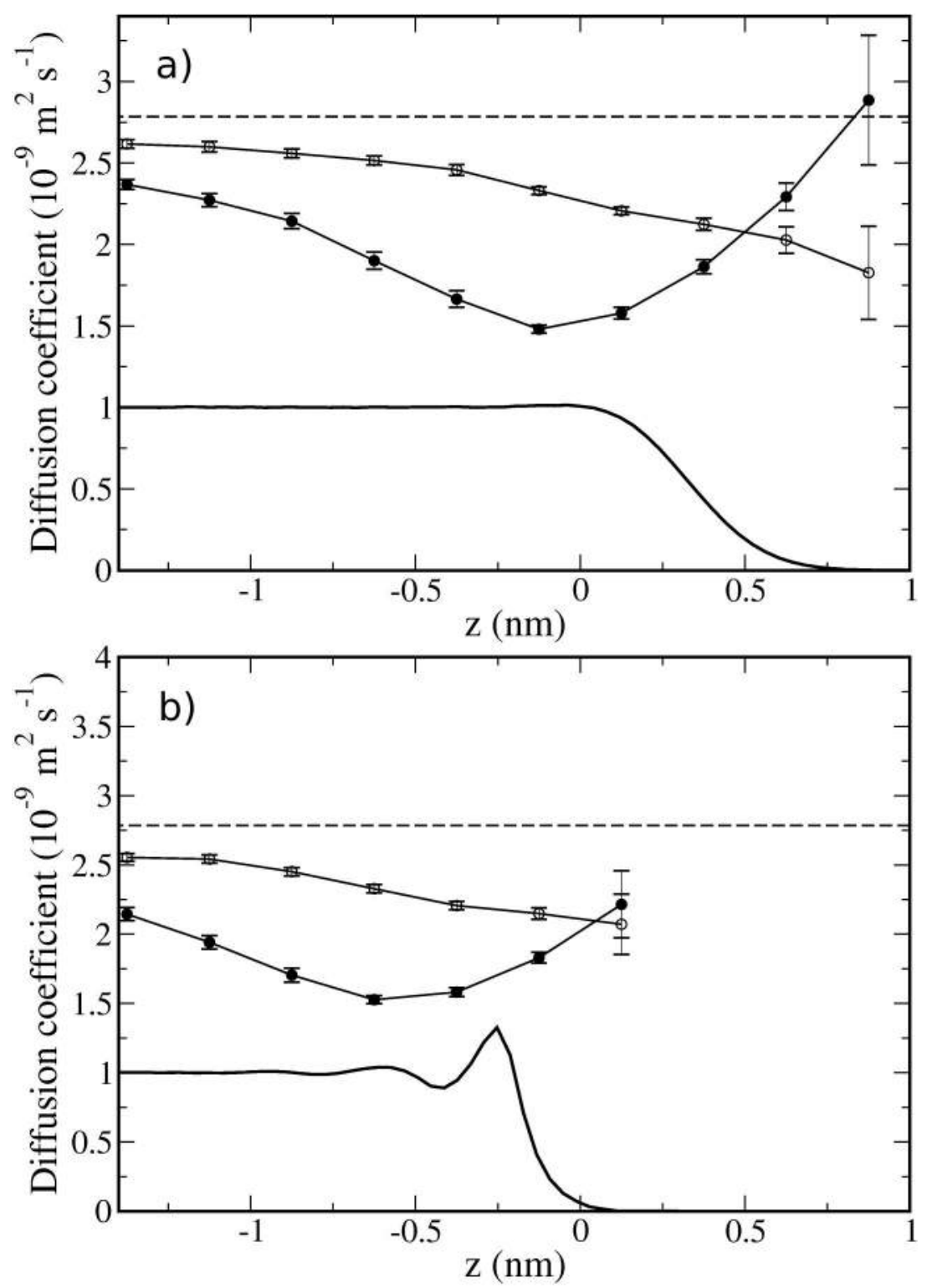

Figure 9. Water diffusion coefficient profiles, global (a) and intrinsic (b), for the water/NPOE interface with $L=4.5 \mathrm{~nm}$. Open symbols are for diffusion in the plane of the interface, closed symbols are for diffusion perpendicular to the interface and the dashed line is the purecomponent diffusion coefficient. The water density profiles (thick lines) are superimposed for ease of visualization. Negative values are within the water phase. 

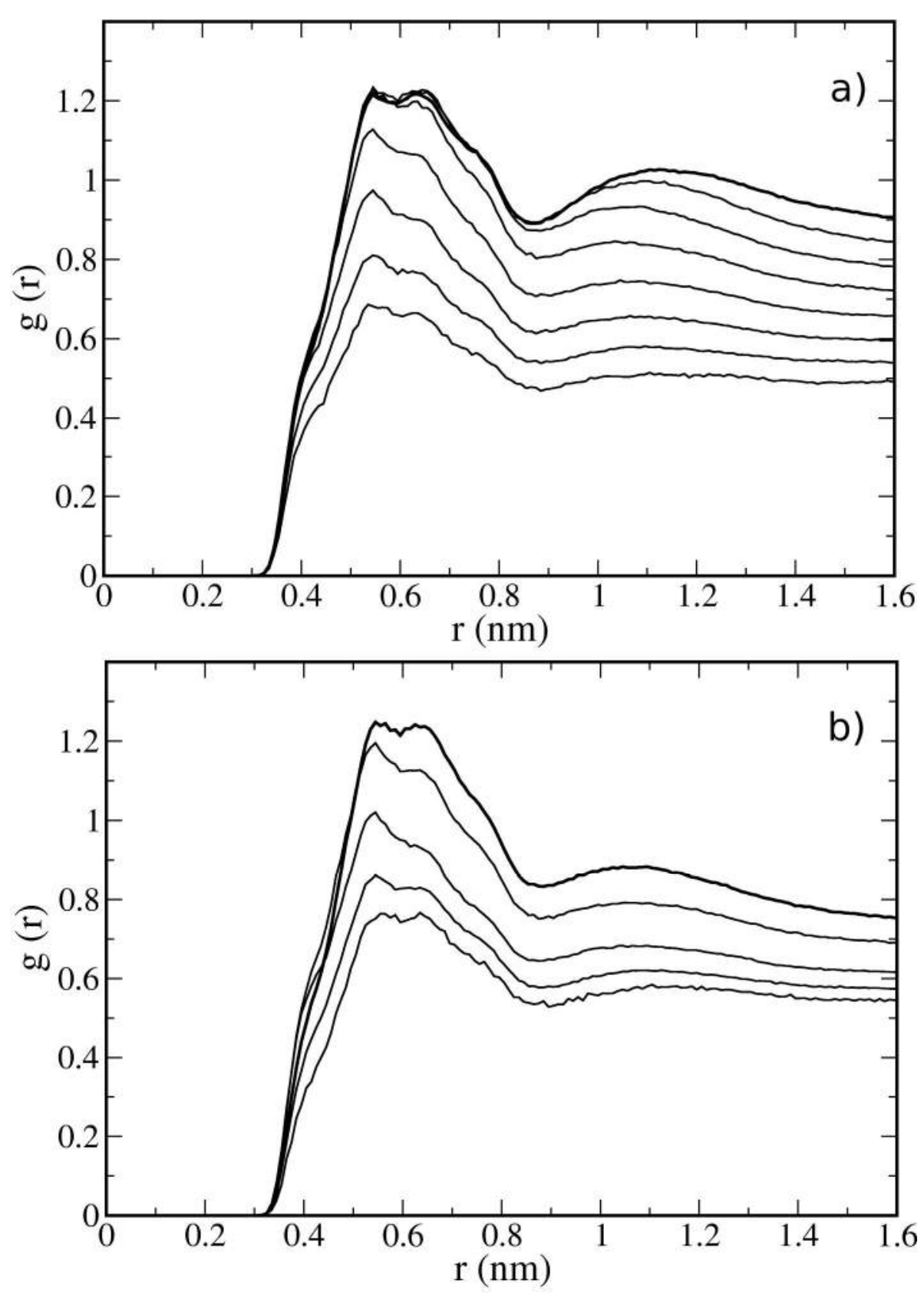

Figure 10. Global (a) and intrinsic (b) $\mathrm{C}_{\mathrm{N}}-\mathrm{C}_{\mathrm{N}}$ RDFs for the system water/NB with $L=3.5 \mathrm{~nm}$ in slices perpendicular to the interface. The curves, from top to bottom, correspond to positions relative to the limit of the water phase of: $0.9,0.7,0.5,0.3,0.1,-0.1,-0.3$, and $-0.5 \mathrm{~nm}$ (the last three curves of part b) are not statistically meaningful). The first curves are highlighted with thick lines for clarity. 

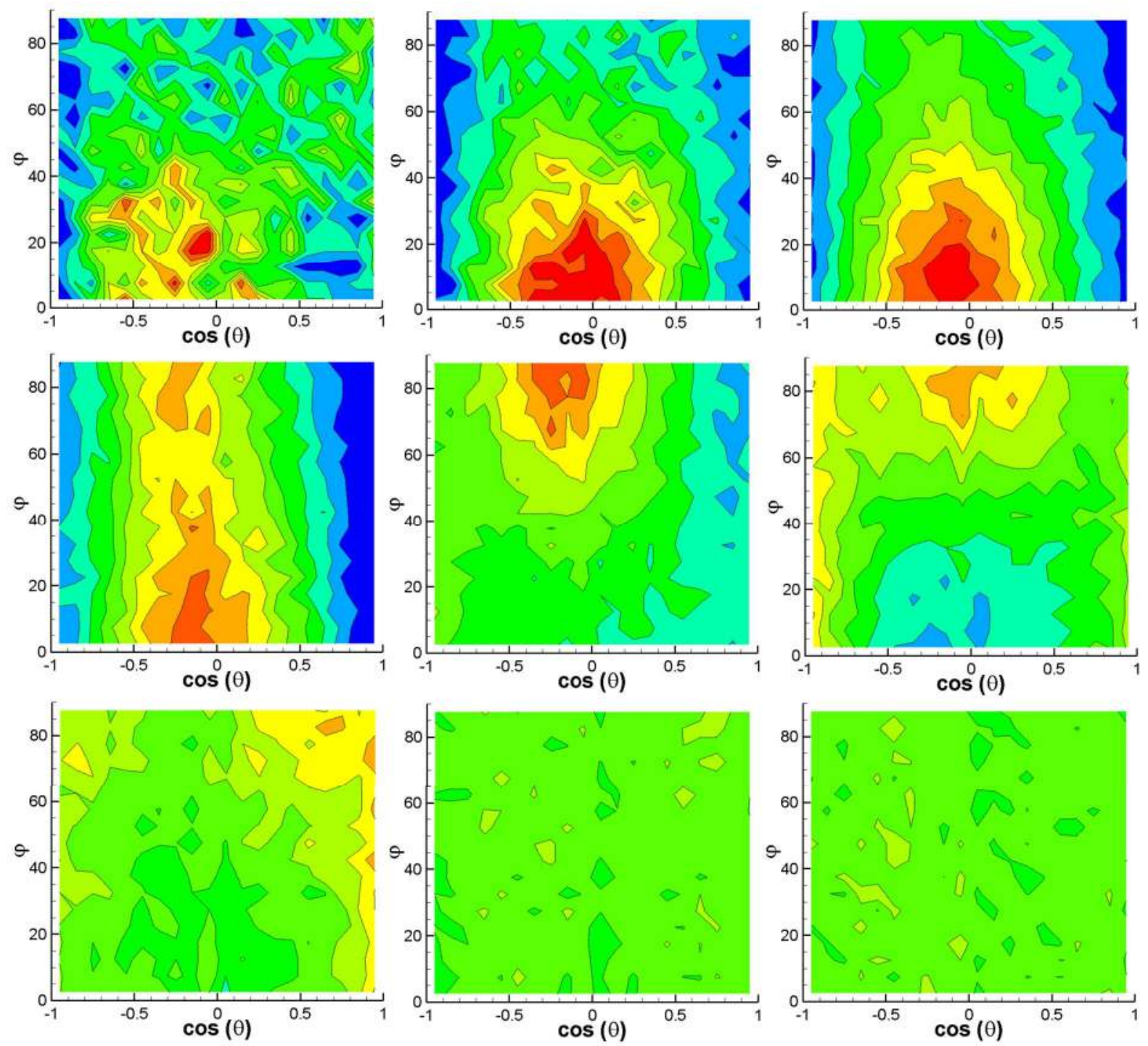

Figure 11. Global orientation distributions of NB molecules for the system water/NB with $L=3.5$ $\mathrm{nm}$ calculated in slices perpendicular to the interface. Slices, from top to bottom, correspond to positions relative to the limit of the organic phase of: $-0.9,-0.7,-0.5,-0.3,-0.1,0.1,0.3,0.5$ and $0.7 \mathrm{~nm}$ (positive values are within the organic phase). Red corresponds to high normalized probability and blue to low probability. 

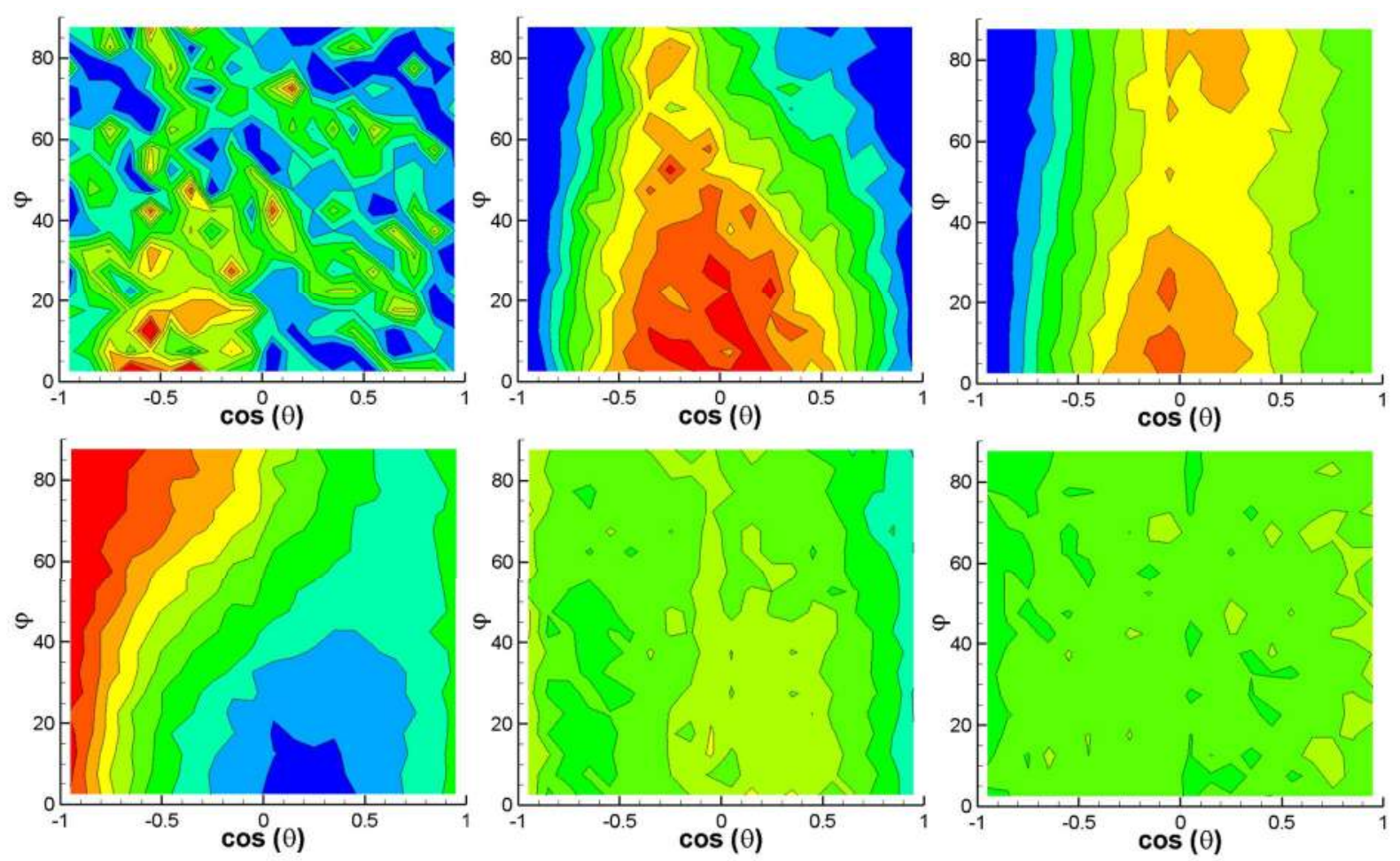

Figure 12. Intrinsic orientation distributions of NB molecules for the system water/NB with $L=3.5 \mathrm{~nm}$ calculated in slices perpendicular to the interface. Slices, from top to bottom, correspond to positions relative to the limit of the organic phase of: $-0.1,0.1,0.3,0.5,0.7$ and 0.9 $\mathrm{nm}$ (slices closer to the water phase are not statistically meaningful). Red corresponds to high normalized probability and blue to low probability. 

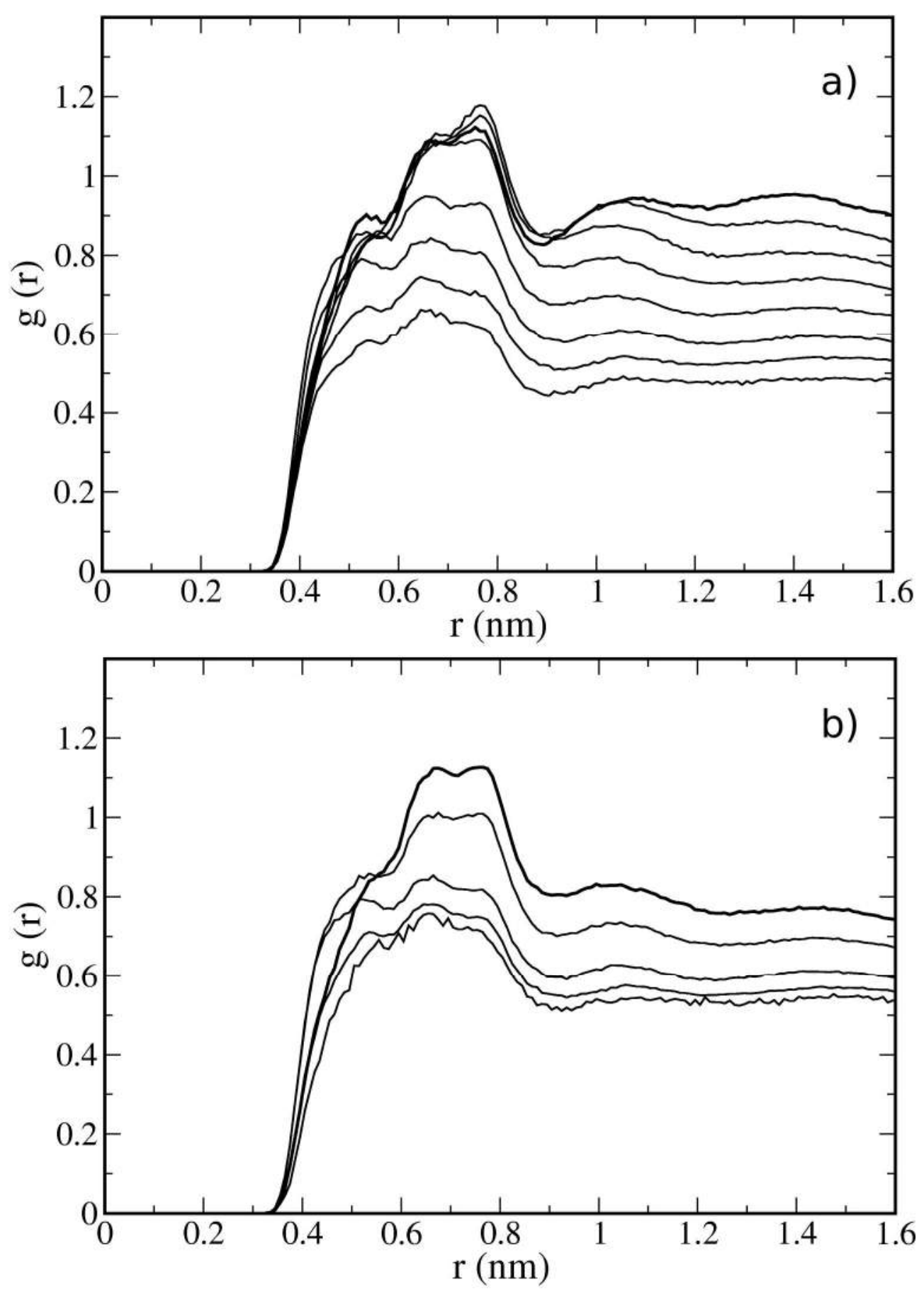

Figure 13. Global (a) and intrinsic (b) $\mathrm{C}_{N}-\mathrm{C}_{\mathrm{N}} \mathrm{RDFs}$ for the system water/NPOE with $L=4.5 \mathrm{~nm}$ in slices perpendicular to the interface. Lines and distances to the limit of the water phase are the same as in Figure 10. 

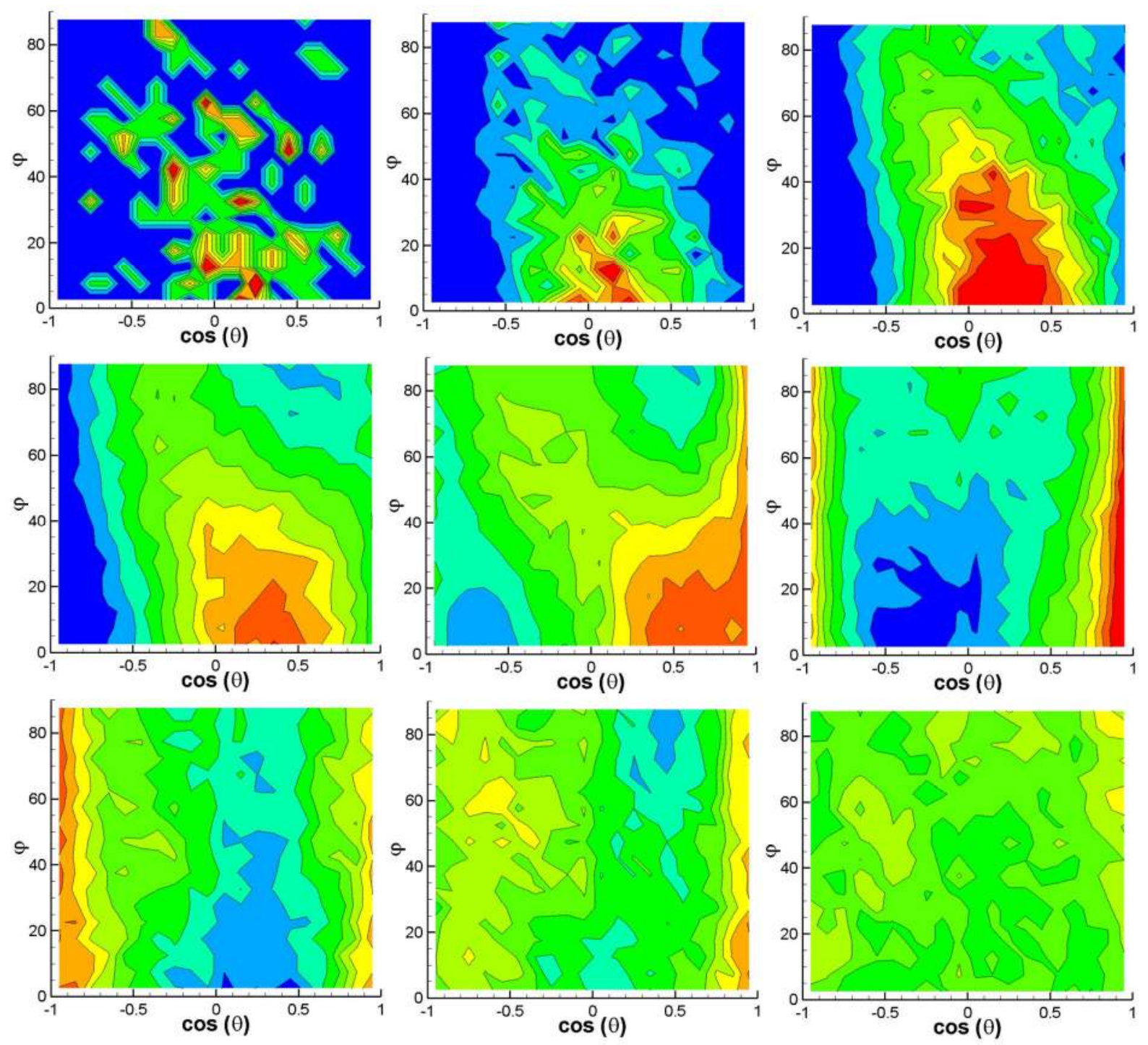

Figure 14. Global orientation distributions of NPOE molecules for the system water/NPOE with $L=3.5 \mathrm{~nm}$ calculated in slices perpendicular to the interface. Colors and distances to the limit of the water phase are the same as in Figure 12. 

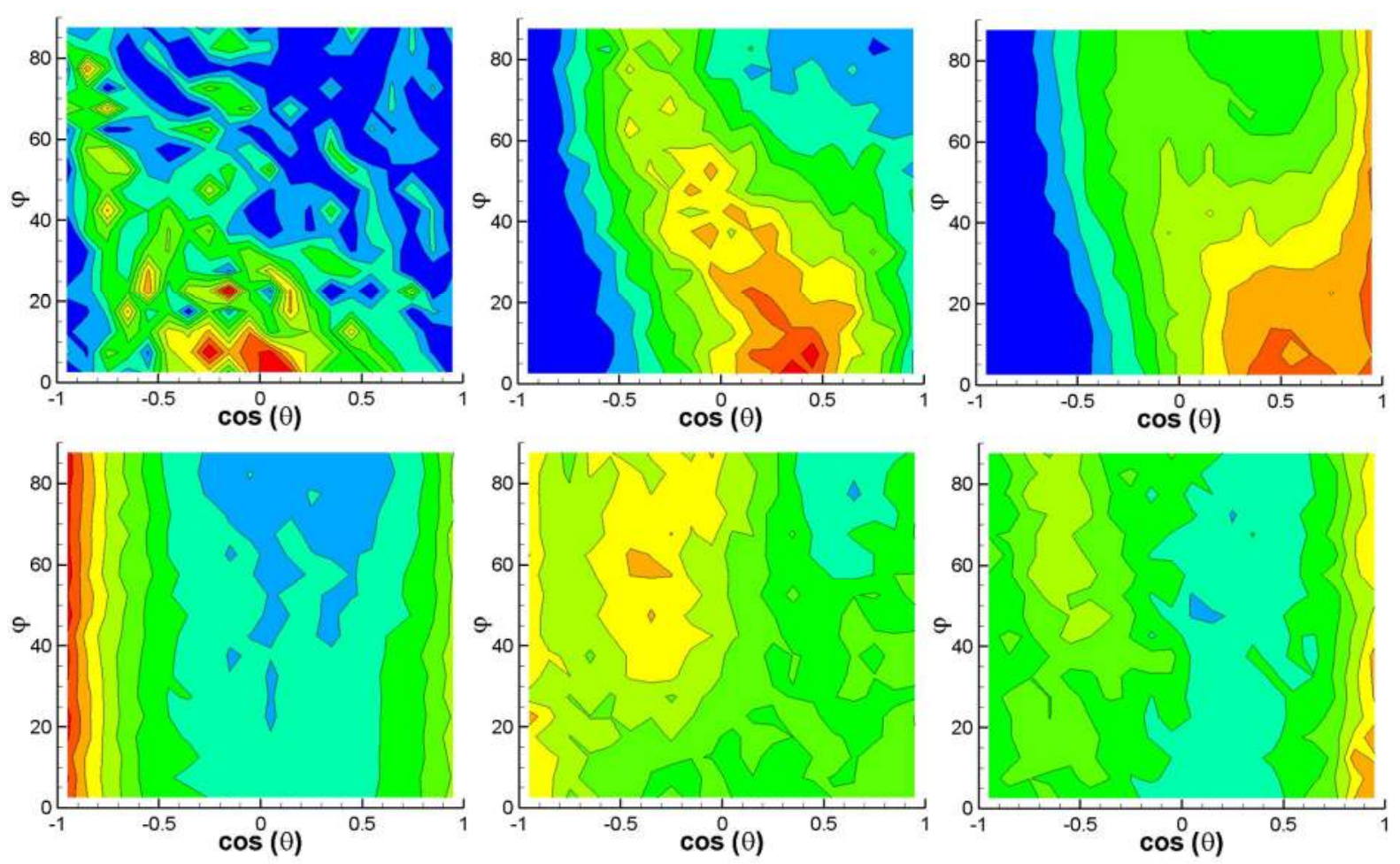

Figure 15. Intrinsic orientation distributions of NPOE molecules for the system water/NPOE with $L=3.5 \mathrm{~nm}$ calculated in slices perpendicular to the interface. Colors and distances to the limit of the water phase are the same as in Figure 13. 

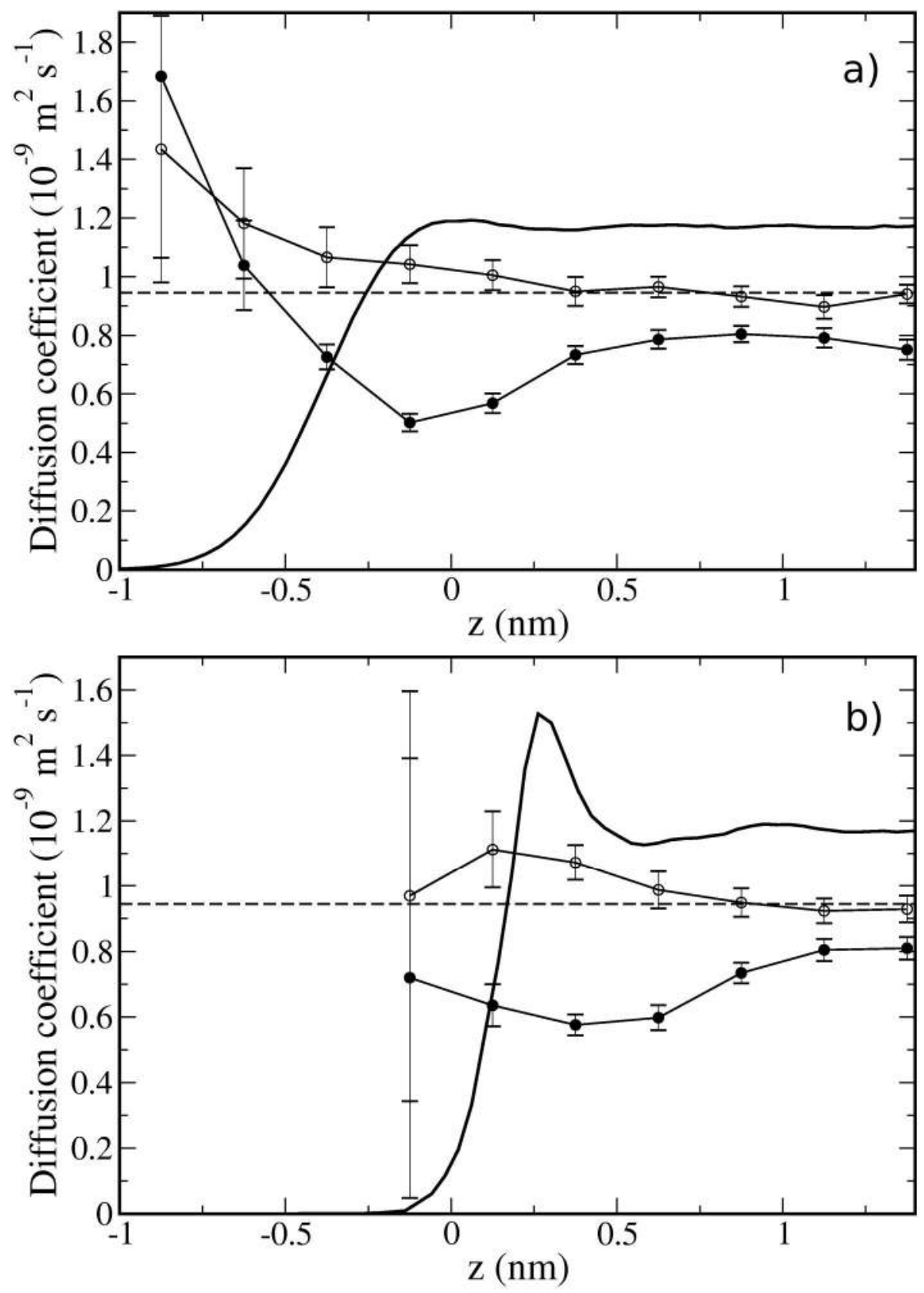

Figure 16. NB diffusion coefficient profiles, global (a) and intrinsic (b) for the system water/NB at $L=3.5 \mathrm{~nm}$. Open symbols are for diffusion in the plane of the interface, closed symbols are for diffusion perpendicular to the interface and the dashed line is the pure-component diffusion coefficient. The organic density profiles (thick lines) are superimposed for ease of visualization. Positive values are within the organic phase. 

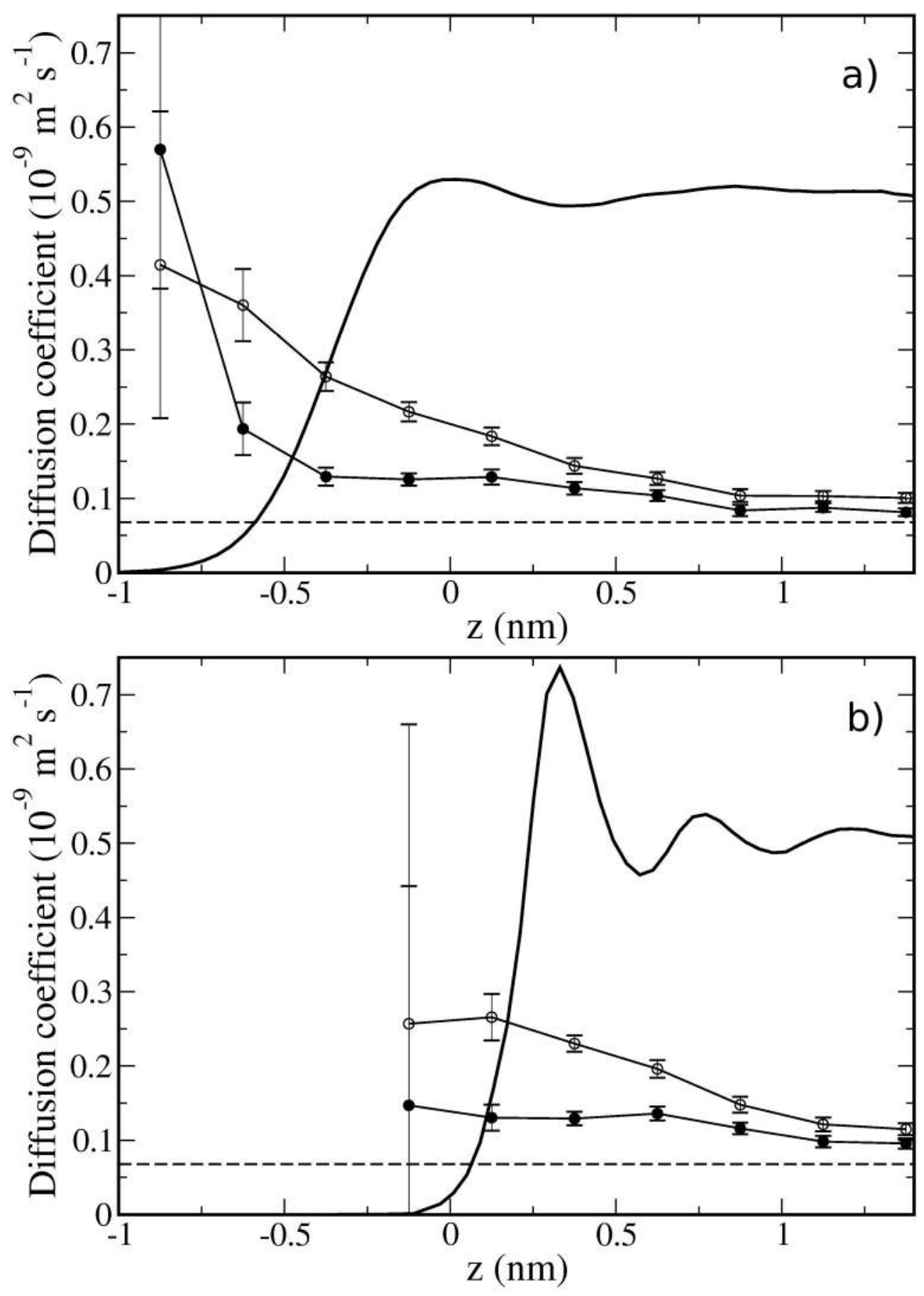

Figure 17. NPOE diffusion coefficient profiles, global (a) and intrinsic (b) for the system water/NPOE at $L=4.5 \mathrm{~nm}$. Lines are the same as in Figure 16. Positive values are within the organic phase. 